\title{
SH2 Domain-Containing Phosphatase-2 Is a Novel Antifibrotic Regulator in Pulmonary Fibrosis
}

\author{
Argyrios Tzouvelekis $^{1 *}$, Guoying Yu ${ }^{1 *}$, Christian L. Lino Cardenas ${ }^{2}$, Jose D. Herazo-Maya ${ }^{1}$, Rong Wang ${ }^{1}$, \\ Tony Woolard ${ }^{1}$, Yi Zhang ${ }^{1}$, Koji Sakamoto ${ }^{1}$, Hojin Lee ${ }^{3}$, Jae-Sung Yi ${ }^{3}$, Giuseppe Deluliis ${ }^{1}$, Nikolaos Xylourgidis ${ }^{1}$, \\ Farida Ahangari ${ }^{1}$, Patty J. Lee ${ }^{1}$, Vassilis Aidinis ${ }^{4}$, Erica L. Herzog ${ }^{1}$, Robert Homer ${ }^{5}$, Anton M. Bennett ${ }^{3}$, and \\ Naftali Kaminski $i^{1}$ \\ ${ }^{1}$ Section of Pulmonary, Critical Care and Sleep Medicine, Department of Internal Medicine, Yale School of Medicine, New Haven, \\ Connecticut; ${ }^{2}$ Thoracic Aortic Center, Massachusetts General Hospital, Harvard Medical School, Boston, Massachusetts; ${ }^{3}$ Department \\ of Pharmacology, Yale School of Medicine, New Haven, Connecticut; "Biomedical Sciences Research Center "Alexander Fleming," Vari, \\ Athens, Greece; and ${ }^{5}$ Department of Pathology, Yale School of Medicine, New Haven, Connecticut
}

ORCID ID: 0000-0001-5917-4601 (N.K.).

\section{Abstract}

Rationale: Idiopathic pulmonary fibrosis (IPF) is a chronic fatal lung disease with dismal prognosis and no cure. The potential role of the ubiquitously expressed $\mathrm{SH} 2$ domain-containing tyrosine phosphatase2 (SHP2) as a therapeutic target has not been studied in IPF.

Objectives: To determine the expression, mechanistic role, and potential therapeutic usefulness of SHP2 in pulmonary fibrosis.

Methods: The effects of SHP2 overexpression and inhibition on fibroblast response to profibrotic stimuli were analyzed in vitro in primary human and mouse lung fibroblasts. In vivo therapeutic effects were assessed in the bleomycin model of lung fibrosis by SHP2-lentiviral administration and transgenic mice carrying a constitutively active SHP2 mutation.

Measurements and Main Results: SHP2 was down-regulated in lungs and lung fibroblasts obtained from patients with IPF. Immunolocalization studies revealed that SHP2 was absent within fibroblastic foci. Loss of SHP2 expression or activity was sufficient to induce fibroblast-to-myofibroblast differentiation in primary human lung fibroblasts. Overexpression of constitutively active SHP2 reduced the responsiveness of fibroblasts to profibrotic stimuli, including significant reductions in cell survival and myofibroblast differentiation. SHP2 effects were mediated through deactivation of fibrosis-relevant tyrosine kinase and serine/threonine kinase signaling pathways. Mice carrying the Noonan syndrome-associated gain-of-function SHP2 mutation $\left(\mathrm{SHP} 2^{\mathrm{D} 61 \mathrm{G} /+}\right)$ were resistant to bleomycin-induced pulmonary fibrosis. Restoration of SHP2 levels in vivo through lentiviral delivery blunted bleomycin-induced pulmonary fibrosis.

Conclusions: Our data suggest that SHP2 is an important regulator of fibroblast differentiation, and its loss as observed in IPF facilitates profibrotic phenotypic changes. Augmentation of SHP2 activity or expression should be investigated as a novel therapeutic strategy for IPF.

Keywords: lung fibrosis; SHP2; PTPN11; antifibrotic therapy

(Received in original form February 16, 2016; accepted in final form September 1, 2016)

${ }^{*}$ A.T. and G.Y. contributed equally to this work.

Supported by National Institutes of Health grants R01 HL095397, U01HL108642, RC2 HL101715, R01 HL127349, P01 DK57751, R01 AR066003, HL109233, and HL125850; American Lung Association grant RT-350419; and Marie Curie ERS/EU-RESPIRE 2 grant 8860/2015.

Author Contributions: A.T. and G.Y. performed most of the experiments with the assistance of C.L.L.C., J.D.H.-M., R.W., T.W., J.-S.Y., G.D., K.S., N.X., and F.A. H.L. performed immune complex phosphatase assays. Y.Z. constructed the lentivectors. R.H. helped with interpretation of some of the immunohistochemistry images. A.T., N.K., and A.M.B. designed the research project and wrote the article. E.L.H., P.J.L., and V.A. revised the article for important intellectual content.

Correspondence and requests for reprints should be addressed to Naftali Kaminski, M.D., Pulmonary, Critical Care and Sleep Medicine, Yale School of Medicine, 300 Cedar Street, TAC-441 South, New Haven, CT 06520-8057. E-mail: naftali.kaminski@yale.edu

This article has an online supplement, which is accessible from this issue's table of contents at www.atsjournals.org.

Am J Respir Crit Care Med Vol 195, Iss 4, pp 500-514, Feb 15, 2017

Copyright $\odot 2017$ by the American Thoracic Society

Originally Published in Press as DOI: 10.1164/rccm.201602-03290C on October 13, 2016

Internet address: www.atsjournals.org 


\section{At a Glance Commentary}

\section{Scientific Knowledge on the}

Subject: Idiopathic pulmonary fibrosis (IPF) is a chronic fatal lung disease characterized by aberrant remodeling, accumulation of extracellular matrix, and formation of myofibroblast foci. The role of $\mathrm{SH} 2$ domain-containing tyrosine phosphatase-2 (SHP2), a ubiquitously expressed tyrosine phosphatase, has not been studied in IPF.

\section{What This Study Adds to the}

Field: SHP2 is down-regulated in myofibroblast foci in IPF lungs. In cell culture, decreases in SHP2 induce profibrotic changes in lung fibroblasts, whereas enhanced expression of SHP reverses these changes. In an animal model of pulmonary fibrosis, lentiviral administration of SHP2 to the lung, or expression of a constitutively active SHP2 mutant, blunts fibrosis. Taken together, our results suggest that augmentation of SHP2 expression or activity should be investigated as a novel therapeutic strategy for IPF.

Idiopathic pulmonary fibrosis (IPF) is a devastating chronic lung disease that leads to death within 3-3.5 years in nearly onehalf of patients $(1,2)$. Current pathogenic theories suggest an aberrant woundhealing process in response to repetitive injuries to the alveolar epithelium in genetically predisposed individuals (3-7). The IPF lung is characterized by extensive histological changes that include formation of fibroblast/myofibroblast foci, accumulation of extracellular matrix, and areas of aberrant remodeling interspersed with normal lung parenchyma (8). Two drugs, pirfenidone (9) and nintedanib (10), have been shown to slow lung function decline in IPF. Considering that nintedanib is a small-molecule tyrosine kinase inhibitor with activity against fibroblast growth factor receptor, vascular endothelial growth factor receptor, and plateletderived growth factor receptor (PDGFR), interest in aberrant tyrosine kinase activation in human lung fibrosis has been reinforced (11).

The activation state of tyrosine kinases is usually tightly regulated by protein tyrosine phosphatases (PTPs) $(12,13)$. The PTP family consists of receptor-like and nonreceptor proteins that affect signal transduction by removing phosphate groups from tyrosine residues of tyrosyl phosphorylated substrates. SH2 domain-containing tyrosine phosphatase-2 (SHP2), a ubiquitously expressed nonreceptor PTP containing two Src homology 2 (SH2) domains and one catalytic PTP domain, is encoded by the protein tyrosine phosphatase nonreceptor type 11 (PTPN11) gene (14). Activating mutations in SHP2 underlie the Noonan syndrome that manifests as congenital heart disease, short stature, and facial dysmorphia $(15,16)$. SHP2 regulates activated tyrosine kinase receptors including PDGFR, by binding to and dephosphorylating them $(17,18)$. In mice targeted deletions of SHP2 in alveolar epithelial cells (AECs) (19) or macrophages (20) increase predisposition to fibrosis, but the expression of SHP2 in human pulmonary fibrosis or its potential therapeutic usefulness are still unknown.

When we analyzed SHP2 expression in IPF lungs we discovered that it was moderately expressed in hyperplastic alveolar and bronchiolar epithelium but generally not within areas of interstitial and peribronchial fibrosis. SHP2 inhibition promoted spontaneous fibroblast-tomyofibroblast differentiation in normal human lung fibroblasts (NHLFs), in vitro, and overexpression of SHP2 reduced the responsiveness of fibroblasts to profibrotic stimuli. SHP2 inhibition in vivo augmented bleomycin-induced pulmonary fibrosis, and restoration of SHP2 levels in vivo through lung-targeted lentiviral delivery or expression of a constitutively active mutant of SHP2 blunted bleomycininduced pulmonary fibrosis. Some of the results of these studies have been previously reported in the form of an abstract (21).

\section{Methods}

For details, see the online supplement.

\section{Gene Expression Data}

We obtained gene expression data from the Lung Genomics Research Consortium (LGRC) gene expression data set (available at GSE47460 and the LGRC website [http://www.lung-genomics.org/], and described in References 22-24).

\section{IPF Tissues}

Additional lung tissue samples were surgical remnants of biopsies or lung explants obtained through the University of Pittsburgh Health Sciences Tissue Bank and Yale University Pathology Tissue service. For RNA and protein extraction, see the online supplement.

\section{Cell Culture Reagents, Cells, Expression Vectors, and Knockdown Experiments}

We used empty pIRES-GFP plasmid (mock) and pIRES-GFP expression plasmids encoding wild-type SHP2, constitutively active E76A, and catalytically inactive R465M constructed as described $(25,26)$. For the SHP2 knockdown experiment we used SHP2specific small interfering RNA (siRNA) and nonspecific siRNA as control. Cells were treated with either transforming growth factor (TGF)- $\beta 1$ (10 ng/ml), PDGF-BB (25 ng/ml), or phenylhydrazonopyrazolone sulfonate-1 (PHPS1) (5 or $10 \mu \mathrm{M})$. In most experiments we used commercially obtained NHLFs or primary mouse lung fibroblasts derived by us. For comparison between IPF and control lung fibroblasts we used primary fibroblasts obtained by C. Feghali-Bostwick (Division of Rheumatology and Immunology, Medical University of South Carolina, Charleston, SC) (27).

\section{Immunoprecipitation Phosphatase Assay}

Briefly, SHP2 phosphatase activity assessment was performed by measuring released para-nitrophenyl phosphate (PNPP; New England BioLabs, Ipswich, MA).

\section{Quantitative Real-Time PCR}

TaqMan (Life Technologies, Thermo Scientific Inc., Rockford, IL) was used according to the manufacturer's instructions, using $\beta$-glucuronidase as an internal control and manufacturer's specific primers and probes. Relative gene expression was normalized to a value of 1.0 for the unstimulated control group. Fold change was calculated by taking the mean of the controls as baseline.

\section{Antibodies}

A detailed list of antibodies is provided in the online supplement. 
A

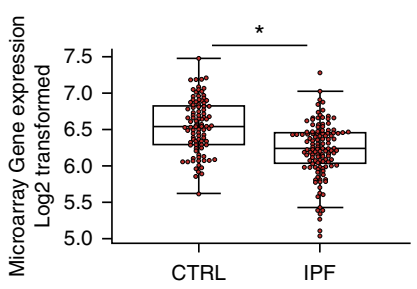

B

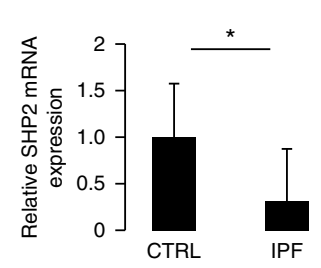

D

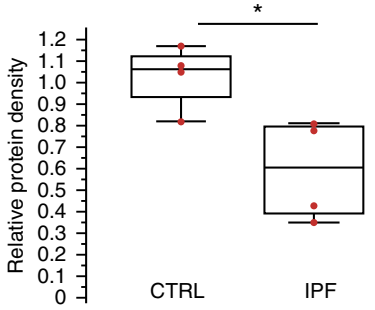

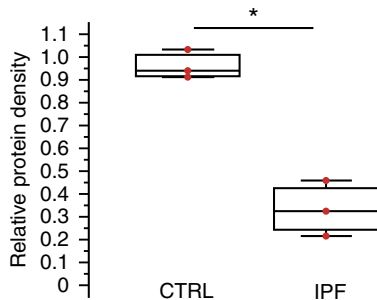

C

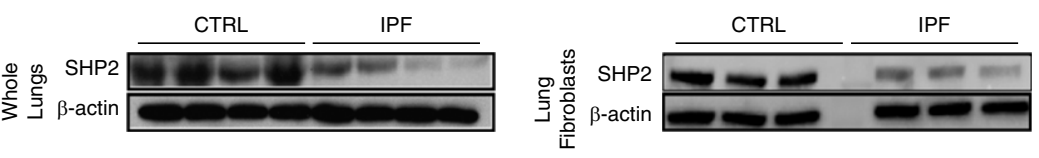

E
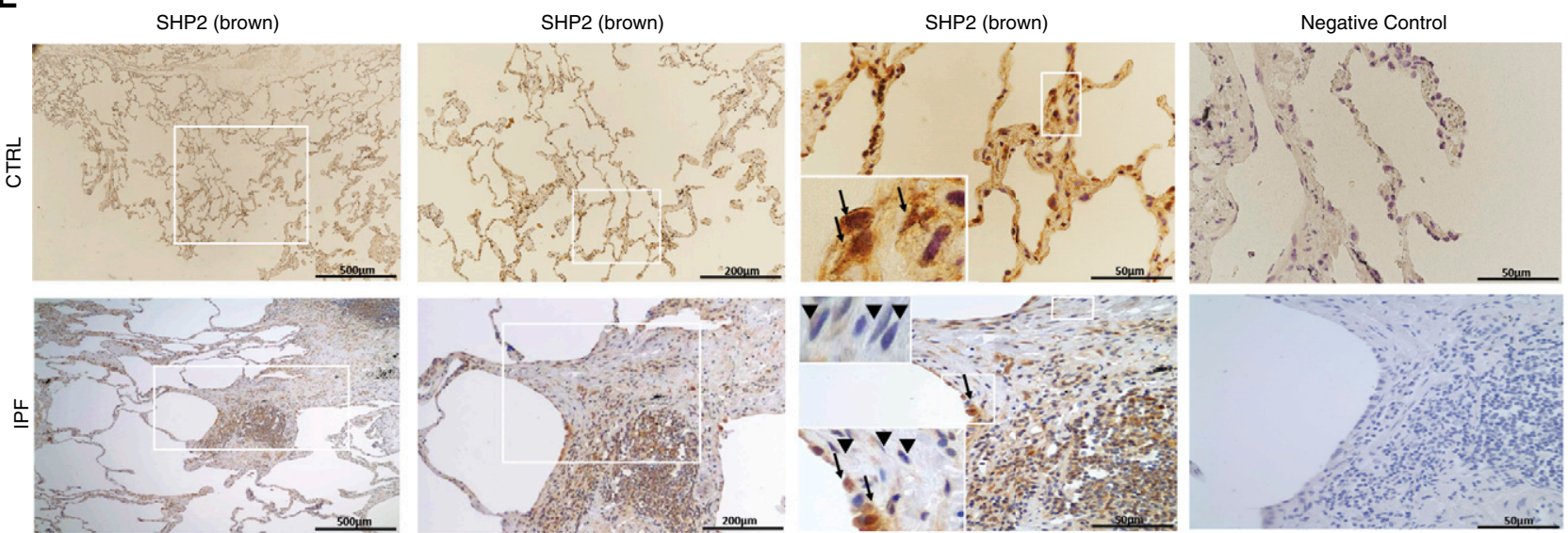

$\mathbf{F}$

SPC (green)/SHP2 (red)/DAPI (blue)/ (merged-yellow)
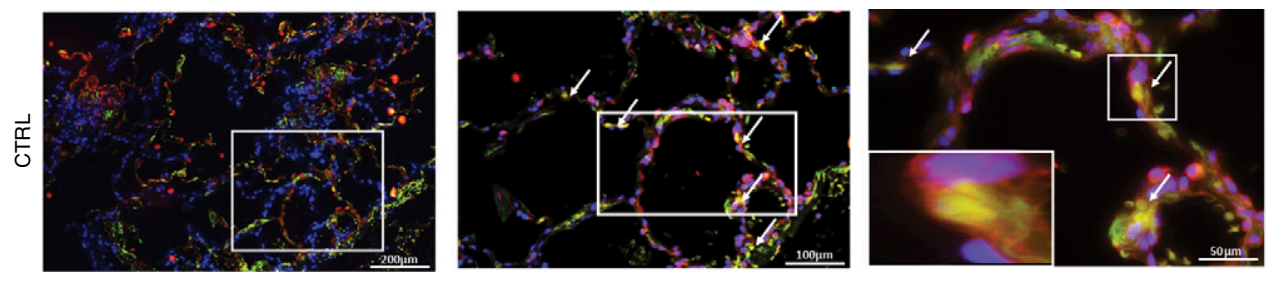

a-SMA (green)/SHP2 (red)/DAPI (blue)/ (merged-yellow)
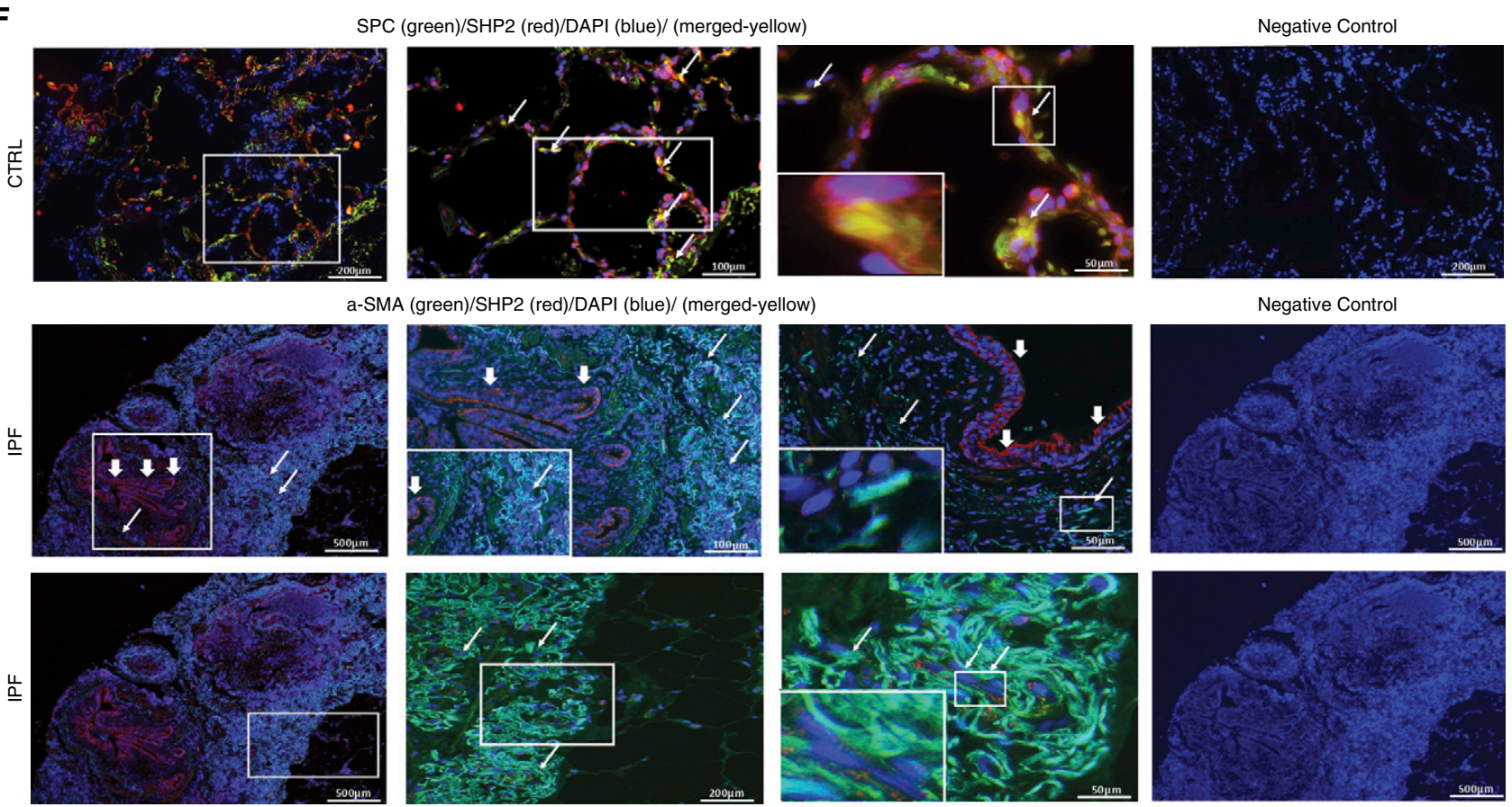

Negative Control
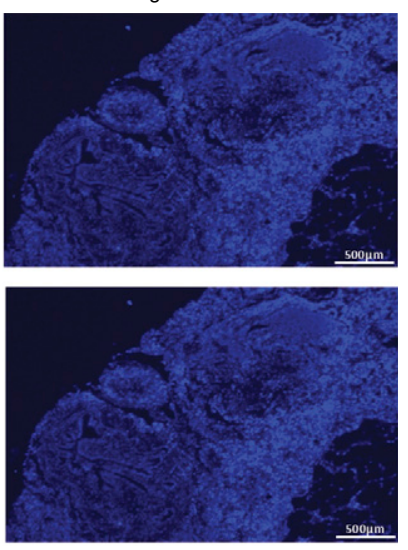

Figure 1. Decreased SH2 domain-containing phosphatase-2 (SHP2) expression in idiopathic pulmonary fibrosis (IPF) lungs and lung fibroblasts. (A) Analysis of the Lung Genomics Research Consortium cohort showed significantly decreased SHP2 expression in patients with IPF ( $\mathrm{n}=123$ ) compared with control (CTRL) subjects $(n=96)\left(6.2\right.$ vs. 6.5, interquartile range [IQR], 6-6.5 vs. 6.3-6.8, respectively; ${ }^{*} P<0.0001$; false discovery rate $\left.<5 \%\right)$. Log $_{2}$-transformed data are presented as box-and-whisker plots, with horizontal bars representing medians, top whisker representing maximal expression, and bottom whisker representing the fifth percentile. Mann-Whitney $U$ test for independent samples. (B) Significant reduction in SHP2 mRNA levels 


\section{Lentivirus Production and Infection}

Lentivirus production, 293T cell infection, and titer measurement were previously described (28).

\section{Mouse Model of Pulmonary Fibrosis \\ All animal studies were conducted in accordance with the National Institutes of Health (Bethesda, MD) guidelines for humane treatment of animals and were approved by the Institutional Animal Care and Use Committee of Yale University (2014-11592, 2013-07867). PHPS1 was administered intraperitoneally $(1.25 \mathrm{mg} / \mathrm{kg})$ on Days 3 $P<0.05$. Microarray statistical analysis was done, in which $\log _{2}$-transformed, cyclic loess-normalized (29) expression values of SHP2 were compared between IPF and control or chronic obstructive pulmonary disease (COPD) samples, using the Mann-Whitney $U$ test for independent samples. A false discovery rate less than $5 \%$ was considered statistically significant.} and 9 after bleomycin $(2.0 \mathrm{U} / \mathrm{kg})$ or normal saline (0.9\%). Experimental groups are detailed in the online supplement. Lenti-wild-type SHP2 or empty lentivector (EV) was intratracheally administered 5 days before bleomycin. Noonan syndrome-associated active SHP2 mutant $\left(S H P 2^{\mathrm{D} 61 \mathrm{G} /+}\right)$ mice are of mixed C57/BL6/SV129 background, so wild-type littermates were used as controls. All experiments used 9- to 12-week-old female C57BL/6 mice unless specified otherwise. Each experiment was performed twice by two independent researchers (A.T., G.Y.) blinded to the identity of the samples. Animals were blindly randomized to receive bleomycin, saline and PHPS1, or lenti-SHP2 or EV.

\section{Assessment of Fibrosis}

Histological analysis, immunohistochemistry, and the hydroxyproline assay are described in the online supplement.

\section{Statistical Analysis}

Data were statistically analyzed with MedCalc version 14 (MedCalc Software, Ostend, Belgium). We used the Mann-Whitney $U$ test or an unpaired $t$ test for comparisons between two groups and one-way analysis of variance for three or more groups. Data are presented as means $\pm \mathrm{SD}$, and considered statistically significant at

\section{Results}

\section{SHP2 Is Decreased in IPF Lungs}

Analysis of LGRC gene expression data revealed that SHP2 was significantly decreased in IPF lungs $(n=123)$ compared with control lungs $(n=96)$ or COPD lungs $(\mathrm{n}=123)$ (Figure 1A; and see Figure E1A and Table E1 in the online supplement). LGRC microarray data were validated by qRT-PCR and immunoblot protein assays revealing statistically significant $(P<0.001)$, 3- and 2.5-fold decreases in SHP2 mRNA and protein (Figure 1B; and Figures $1 \mathrm{C}$ and $1 \mathrm{D}$, top), respectively. Immunoblot analyses showed significantly decreased SHP2 expression in IPF lung fibroblasts compared with control lung fibroblasts (Figures $1 \mathrm{C}$ and $1 \mathrm{D}$, bottom). SHP2 showed moderate expression in the hyperplastic epithelium, was reduced mainly from areas of active fibroblastic foci (Figure 1E, bottom), and did not colocalize with $\alpha$-smooth muscle actin $(\alpha$-SMA) in IPF lungs (Figure $1 \mathrm{~F}$, bottom). In control lungs SHP2 was expressed mainly by alveolar epithelial cells (Figures $1 \mathrm{E}$ and $1 \mathrm{~F}$, and Figures E1B and E1C).

\section{Inhibition of SHP2 Expression or Catalytic Activity Promotes Fibroblast-to-Myofibroblast Differentiation}

Transfection of NHLFs with siRNA reduced the expression of SHP2 mRNA and protein levels by $70 \%$ (Figures $2 \mathrm{~A}$ and $2 \mathrm{~B}$ ). Knockdown of SHP2 expression in NHLFs caused significant increases in $\alpha$-SMA and collagen type I $\alpha 1$ (COL1A1) mRNAs (four- and fivefold, respectively) and protein levels (Figures $2 \mathrm{~A}$ and $2 \mathrm{~B}$, and Figure E2A) compared with controls. Immunofluorescence staining showed increased formation of stress fibers in si-SHP2-treated NHLFs compared with controls (Figure 2C, and Figure E3A), indicating differentiation of fibroblasts into myofibroblasts. To inhibit SHP2 catalytic activity, we treated NHLFs for 60 minutes with PHPS1, a cell-permeable phosphotyrosine mimetic that acts as a reversible, active site-targeting, substratecompetitive inhibitor of the catalytic domain of SHP2 (30). Treatment of NHLFs with PHPS1 significantly increased the mRNA and protein levels of $\alpha$-SMA and COL1A1 (Figures 2D and 2E, and Figure E2B). Immunofluorescence revealed that SHP2 inhibition with PHPS1 caused the formation of stress fibers, characteristic of myofibroblast differentiation (31), similar to those caused by TGF- $\beta_{1}$ (Figure $2 \mathrm{~F}$, and Figure E3B).

\section{SHP2 Effects on Fibroblast Proliferation and Apoptosis}

We performed gain- or loss-of-function experiments using wild-type SHP2, a constitutively active mutant (E76A), or a catalytically inactive mutant (R465M) in NHLFs. Wild type and E76A produced significant but moderate (1.2-fold)

Figure 1. (Continued). (threefold; $\left.{ }^{\star} P<0.0001\right)$, assessed by quantitative RT-PCR, in whole IPF lungs $(\mathrm{n}=24)$ compared with control lungs $(\mathrm{n}=16)$. Bars and error bars (means $\pm \mathrm{SD}$ ) show the relative changes (fold) by setting the indicated control level to 1.0. Independent samples, Student's $t$ test. (C) Immunoblot of whole lung lysates (top) and lung fibroblasts (bottom), showing lower protein levels of SHP2 in IPF compared with control samples. (D) Immunoblot densitometry analysis normalized to $\beta$-actin shows significantly decreased SHP2 protein levels in IPF lungs compared with control lungs (0.6 vs. 1.1 [IQR, 0.4-0.8 vs. 0.9-1.1], respectively) as well as in IPF lung fibroblasts compared with control fibroblasts (0.32 vs. 0.94). Data are presented as box-and-whisker plots, with horizontal bars representing medians. Mann-Whitney $U$ test for independent samples, ${ }^{\star} P<0.05$. (E) Immunohistochemical analysis in representative lung samples $(\mathrm{n}=5)$ showing SHP2 expression in normal alveolar epithelium in control lungs (top, arrows). SHP2 expression was present in the hyperplastic alveolar epithelium surrounding fibroblastic foci in IPF lungs $(n=5)$ (bottom, arrows), whereas it was almost absent in fibroblastlike cells within active fibroblastic foci (bottom, arrowheads). Boxed regions are shown enlarged on the right and as insets on the left. A nonimmune immunoglobulin of the same isotype and concentration as the primary antibody was used as negative control. $(F)$ Double-immunofluorescence analysis in representative lung tissue samples $(n=5)$ showing that SHP2 (red) colocalized (merged yellow, arrows) with surfactant protein (SP)-C-positive cells (green) in control lungs (top). SHP2 did not colocalize with $\alpha$-smooth muscle actin ( $\alpha$-SMA)-positive cells (green, arrows) within areas of peribronchial and interstitial fibrosis (middle and bottom), whereas it was expressed in the surrounding respiratory epithelium (middle, thick arrows) in IPF lungs. Boxed regions are shown enlarged on the right and as insets on the left. Samples that were incubated without the primary antibodies (SHP2 and SP-C or $\alpha$-SMA) were used as negative controls. 
A

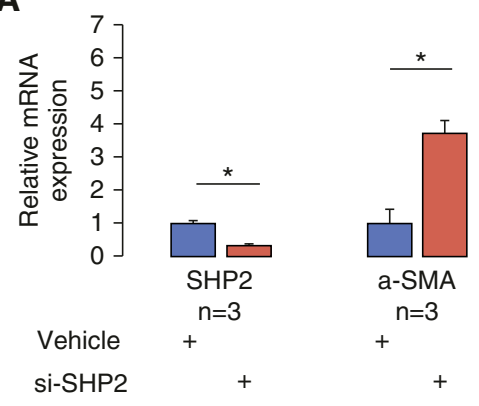

B

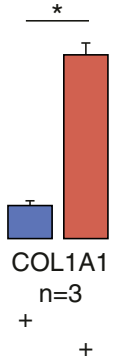

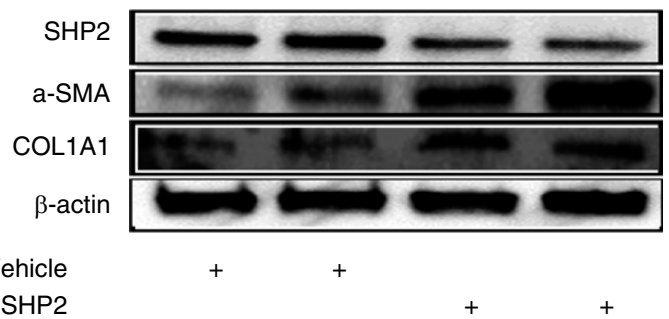

\section{C}

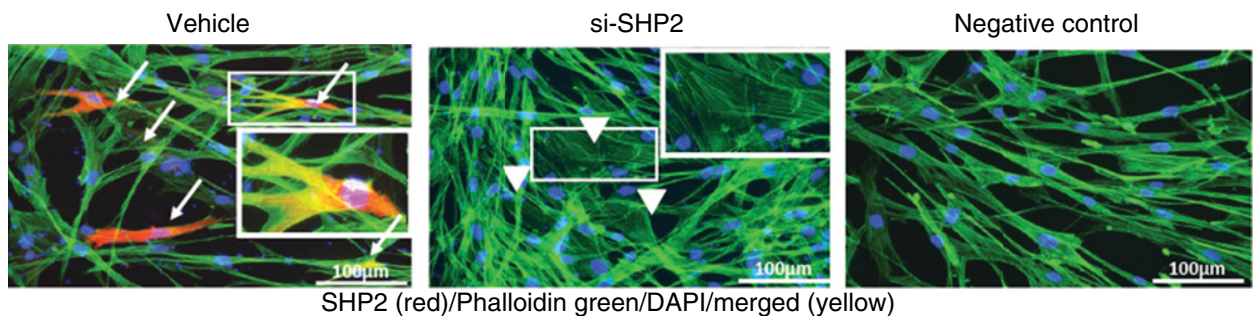

D

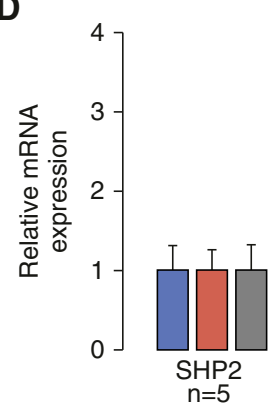

E

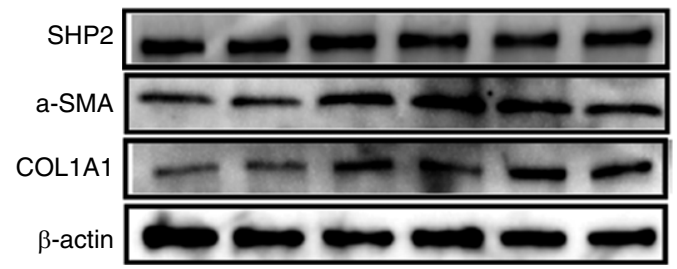

PHPS1-0 $\mu \mathrm{M}++$

PHPS1-5 $\mu \mathrm{M}$

PHPS1-10 $\mu \mathrm{M}$

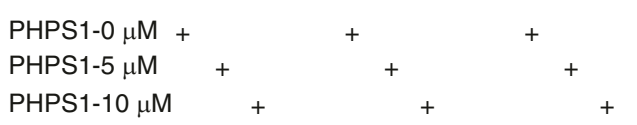

$\mathbf{F}$

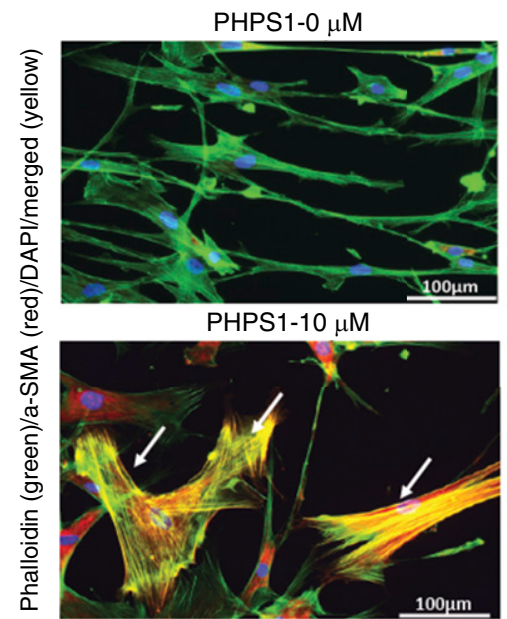

PHPS1-5 $\mu \mathrm{M}$

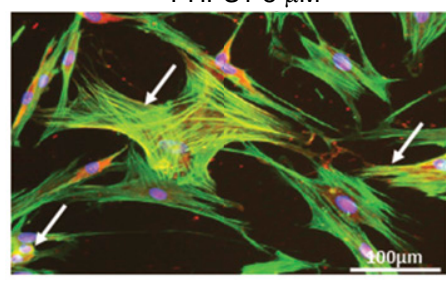

TGFB1

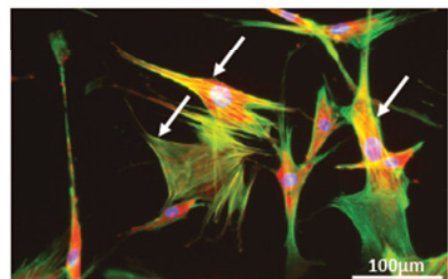

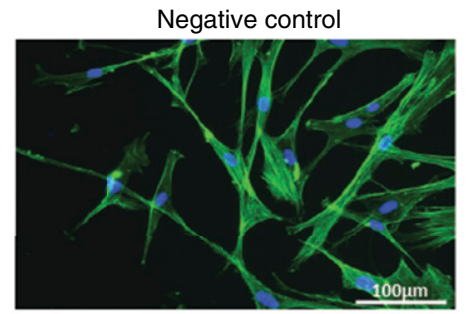

Negative control

Figure 2. SH2 domain-containing phosphatase-2 (SHP2) inhibition promotes fibroblast-to-myofibroblast differentiation. An SHP2-specific small interfering RNA (siRNA) (si-SHP2, $25 \mathrm{nM}$ for $48 \mathrm{~h}$ ) or phenylhydrazonopyrazolone sulfonate 1 (PHPS1), a relatively selective, pharmacological SHP2 inhibitor (0 [equivalent volume of dimethyl sulfoxide], 5, or $10 \mu \mathrm{M}$, for $60 \mathrm{~min}$ ), was used to knock down SHP2 expression or to inhibit the catalytic domain of SHP2, respectively, in normal human lung fibroblasts (NHLFs). Cells were harvested 48 hours afterward for RNA and protein extraction, respectively.

(A) Relative change in SHP2, $\alpha$-smooth muscle actin ( $\alpha$-SMA), and collagen type I $\alpha 1$ (COL1A1) mRNA levels after treatment with si-SHP2 or a nonspecific 
reductions in PDGF-BB-induced proliferation rates of NHLFs compared with cells transfected with empty plasmid (mock treatment) or with R465M (Figure 3A). Both the wild-type SHP2 and E76A mutant caused significant increases in the mRNA and protein levels of caspase- 3 and cleaved caspase-3 (c-CASP3), respectively (Figures $3 \mathrm{~B}$ and $3 \mathrm{C}$, and Figure E2C); however, they had only moderate effects on B-cell CLL/lymphoma 2 (BCL2) levels. SHP2-mediated fibroblast apoptosis was further confirmed by upregulation of caspase- 3 and caspase- 7 activity (6-fold) (Figure 3D) and increased numbers of TUNEL (terminal deoxynucleotidyltransferase dUTP nick end labeling)-positive cells (19-fold) (Figures $3 \mathrm{E}$ and $3 \mathrm{~F}$ ), in NHLFs transfected with wild-type or E76A. As expected, the wild-type- or E76A-transfected NHLFs exhibited higher basal protein tyrosine phosphatase (PTP) activity compared with mock- or R465M inactive mutant-transfected cells (Figure 3G). These observations support a PTP activity-dependent role for SHP2 in regulating fibroblast survival.

\section{SHP2 Is Down-regulated by TGF- $\beta_{1}$ and Its Overexpression Negatively Regulates TGF- $\beta_{1}$-induced Differentiation of Fibroblasts to Myofibroblasts In Vitro}

Stimulation of NHLFs with TGF- $\beta_{1}(10$ $\mathrm{ng} / \mathrm{ml}$ for $6 \mathrm{~h}$ ) significantly decreased SHP2 mRNA (threefold) and protein expression and increased $\alpha$-SMA (twofold) and COL1A1 (1.5-fold) expression (Figures 4A and $4 \mathrm{~B}$, and Figure E2D). Transfection of NHLFs with wild-type or E76A mutant blunted the TGF- $\beta_{1}$-induced increases in $\alpha$-SMA and COL1A1 (Figures $4 \mathrm{~A}$ and $4 \mathrm{~B}$ ), but transfection with $\mathrm{R} 465 \mathrm{M}$ mutant did not. Similarly, transfection of NHLFs with wild type or E76A prevented the TGF- $\beta_{1}$ morphological changes (Figure $4 \mathrm{C}$, and Figure E4), and sustained SHP2 levels. Transfection with R465M did not prevent the changes in cell morphology, despite maintenance of SHP2 levels. Taken together, these observations suggest that regulation of fibroblast-to-myofibroblast differentiation requires an active SHP2 catalytic domain.

\section{SHP2 Negatively Regulates Phosphorylation States of Tyrosine Kinase and Serine/Threonine Kinase Fibrotic Pathways}

In line with previous reports showing dephosphorylation of PDGFR by SHP2 (32), both wild type and E76A reduced the PDGF-BB-induced phosphorylation of c-Abl (site 412), whereas R465M had no effect on PDGF-BB-induced activation of $\mathrm{c}-\mathrm{Abl}$ (Figure 5A, and Figure E2E). Similarly, transfection with wild type and E765A suppressed TGF- $\beta_{1}$-induced Smad $2 / 3$ phosphorylation (key regulatory site 467 and sites 423 and 425, respectively), whereas $\mathrm{R} 465 \mathrm{M}$ or mock treatment did not (Figures $5 \mathrm{~B}$ and $5 \mathrm{C}$, and Figures E2F and E2G).

\section{SHP2 Inhibition Enhances Fibrotic Responses in the Bleomycin Model of Pulmonary Fibrosis}

We investigated the effects of systemic inhibition of SHP2 catalytic activity, using PHPS1 given intraperitoneally $(1.25 \mathrm{mg} / \mathrm{kg})$ on Days 3 and 9 after bleomycin administration (Day 0). PHPS1 increased lung fibrosis both spontaneously and after bleomycin treatment, as indicated by significant increases in hydroxyproline levels (1.5- and 1.3-fold, respectively) and Masson's trichrome and $\alpha$-SMA staining
(Figures 6B and 6C), with no effect on SHP2 mRNA (Figure 6A).

\section{Lentiviral Administration of SHP2 Attenuates Bleomycin-induced Lung Fibrosis}

SHP2 expression was significantly decreased after bleomycin administration (Figure E5A). We designed a lentiviral SHP2 expression vector to determine whether SHP2 overexpression will have an effect on pulmonary fibrosis. The wild-type SHP2 sequence was cloned into a lentiviral vector (Figure E5B) and mice were intratracheally infected with either SHP2-lentivector or EV 5 days before bleomycin or saline administration and killed on Day 14 (19 d after viral administration). Study design was based on pilot SHP2 expression kinetics experiments showing increased lung mRNA expression of SHP2 21 days after SHP2lentiviral administration (Figures E6A and E6B). Infection efficiency was confirmed by significant increases in SHP2 mRNA and protein levels (three- and twofold, respectively; $P<0.05$ ) after SHP2-lentiviral administration compared with the EV-treated and saline-challenged animals (Figures 7A-7C). A significant threefold increase in SHP2 mRNA level was also noted in bleomycin-challenged mice treated with SHP2-lentivector compared with EV-treated animals. A trend toward an increase in SHP2 protein levels (threefold) after SHP2-lentiviral delivery, compared with EV-treated animals, was also observed in bleomycin-challenged animals; however, it did not reach statistical significance, a finding expected because only a small number of samples was available for protein analysis. Interestingly, SHP2 protein levels were inversely correlated to hydroxyproline levels $(r=-0.68, P<0.05$; Figure E6C), further supporting an

Figure 2. (Continued). siRNA construct (empty vector). Each bar represents the mean expression of three NHLF samples (in duplicate). Bars and error bars (means \pm SD) are shown for the relative changes (fold) by setting the indicated control level to 1.0. One-way analysis of variance $(A N O V A),{ }^{*} P<0.05$. (B) Immunoblot analyses of SHP2, $\alpha$-SMA, and COL1A1. Each lane represents an individual NHLF preparation (biological replicates). Blots were stripped and reblotted, using an anti- $\beta$-actin antibody as loading control. (C) Double-immunofluorescence analysis showing colocalization (merged yellow, arrows, inset) of SHP2 with filamentous actin (F-actin, green) in vehicle-treated NHLFs (left). Treatment of NHLFs with siRNA was associated with absence of SHP2 expression and increased formation of stress fibers as indicated by phalloidin green (arrowheads, inset) (middle). Boxed regions are shown as insets on the right. Samples that were incubated without the primary antibody (SHP2) were used as negative controls (right). DAPI = 4',6-diamidino-2phenylindole. (D) Relative change in $\alpha$-SMA and COL1A1 mRNA levels after treatment with 0,5 , and $10 \mu \mathrm{M}$ PHPS1. Each bar and error bar represents the mean $\pm \mathrm{SD}$ expression of five samples (biological replicates). Error bars are shown for the relative changes (fold) by setting the indicated control leve to 1.0. One-way ANOVA, ${ }^{\star} P<0.05$. (E) Immunoblot analyses of SHP2, $\alpha-S M A$, and COL1A1. Each lane represents an individual NHLF preparation (biological replicates). Blots were stripped and reblotted, using an anti- $\beta$-actin antibody as loading control. ( $F$ ) Double-immunofluorescence analysis showing increased fibroblast-to-myofibroblast differentiation as indicated by colocalization (merged yellow, arrows) of $\alpha$-SMA (red) with F-actin (phalloidin green, stress fibers) in PHPS1-treated cells compared with untreated cells (PHPS1, $0 \mu \mathrm{M}$ ). Transforming growth factor (TGF)- $\beta_{1}$ stimulation (10 ng/ml, $360 \mathrm{~min}$ ) was used as positive control. Samples that were incubated without the primary antibody ( $\alpha$-SMA) were used as negative controls. 
A

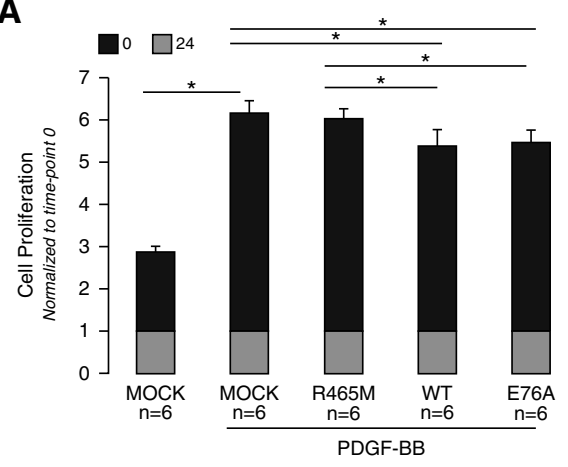

C

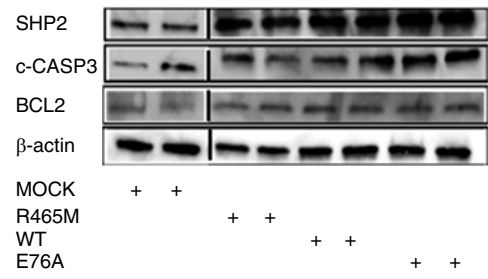

E
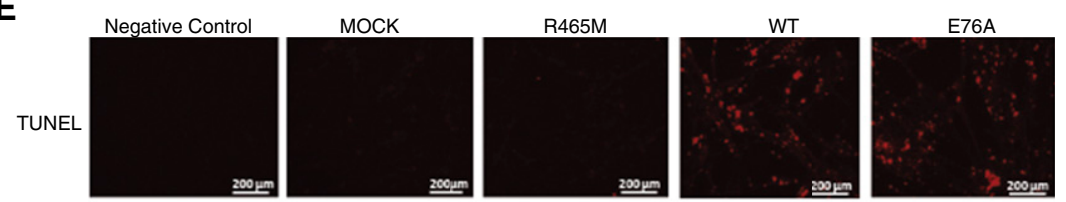

DAPI
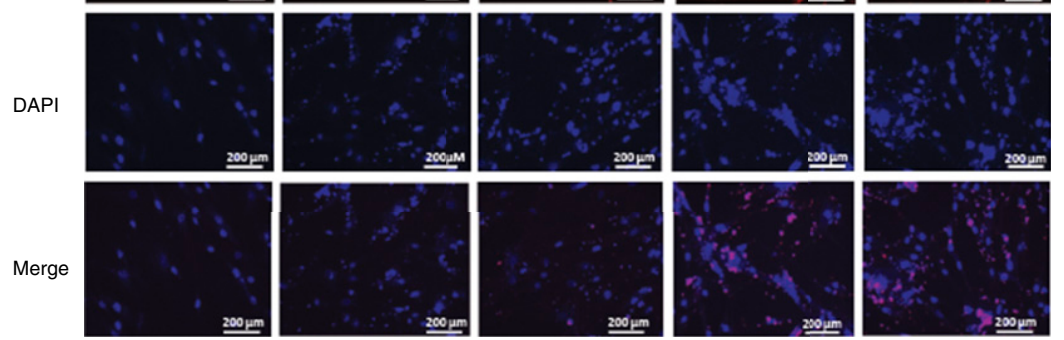

$\mathbf{F}$

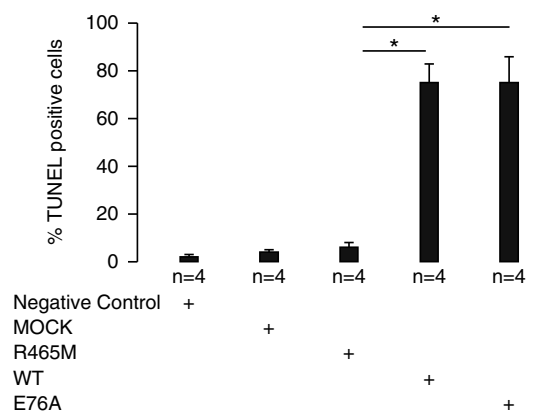

G

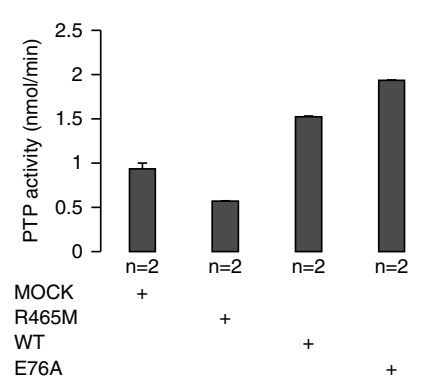

Figure 3. SH2 domain-containing phosphatase-2 (SHP2) negatively regulates fibroblast proliferation and survival in vitro. (A) Normal human lung fibroblasts (NHLFs) were stimulated with platelet-derived growth factor (PDGF)-BB ( $25 \mathrm{ng} / \mathrm{ml}$ ) for 6 hours. PDGF-BB stimulation induced a significant increase (2.7-fold) in proliferation rates of mock- and inactive SHP2 (R465M)-treated cells compared with unstimulated cells. Transfection with active SHP2 (wild-type [WT] and E76A) led to a significant association between reduction in lung SHP2 and increased experimental lung fibrosis. Immunohistochemical analysis suggested SHP2 overexpression in both the epithelial and stromal lung compartments in SHP2-treated animals compared with the EV-treated littermates (Figure E6D). Lentiviral restoration of SHP2 significantly blunted increases in hydroxyproline levels (1.35-fold) (Figure 7D); COL1A1, $\alpha$-SMA, and fibronectin-1 (FN1) mRNAs (2.1-, 1.7-, and 2.2-fold, respectively) (Figure 7E); but not COL3A1 expression levels (1.5-fold; not significant) (Figure 7E).

Immunohistochemistry showed a substantial decrease in Masson's trichrome and $\alpha$-SMA staining in mice treated with SHP2-lentivector compared with $\mathrm{EV}$-treated mice (Figure 7F, top and bottom, respectively).

The Noonan Syndrome-associated SHP2 Constitutively Active Mutant $\left(\mathrm{SHP2}^{\mathrm{D61G} /+}\right.$ ) Confers Resistance to Bleomycin-induced Lung Fibrosis A mouse model bearing the Noonan syndrome common SHP2 gainof-function mutation (aspartate-toglycine substitution in amino acid 61; D61G), which renders the catalytic domain of SHP2 constitutively active, has been shown to faithfully recapitulate the human features of Noonan syndrome, but little is known of its lung phenotype (33). We challenged heterozygous gain-offunction SHP2 mutants $\left(S H P 2^{\mathrm{D} 61 \mathrm{G} /+}\right)$ and wild-type littermates $\left(S H P 2^{+/+}\right)$with bleomycin $(3.0 \mathrm{U} / \mathrm{kg})$ or normal saline. $\mathrm{SHP} 2^{\mathrm{D} 61 \mathrm{G} /+}$ mice were relatively resistant to bleomycin-induced lung fibrosis as indicated by significant reductions in hydroxyproline levels (1.4-fold) (Figure 8A); mRNA levels of COL1A1 (2.9-fold), COL3A1 (3.3-fold), $\alpha$-SMA (1.9-fold), and fibronectin (4.1-fold) (Figure 8B); as well as Masson's trichrome and $\alpha$-SMA staining (Figure 8C, top and bottom, respectively).

\section{Primary Mouse Lung Fibroblasts Expressing the Noonan} Syndrome-associated SHP2 Active Mutant (SHP2 ${ }^{\mathrm{D61G} /+}$ ) Display Attenuated Fibrotic Responses In Vitro We isolated primary mouse lung fibroblasts (MLFs) from $S H P 2^{\mathrm{D} 61 \mathrm{G} /+}$ and $\mathrm{SHP} 2^{+/+}$ mice and assessed their responsiveness to 
A

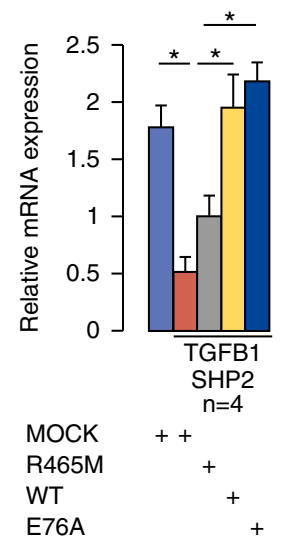

B

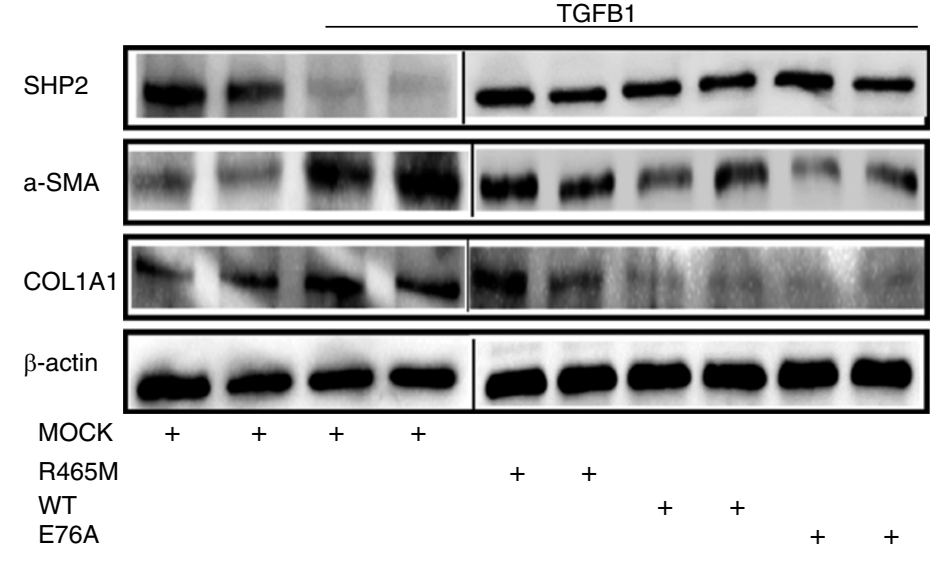

\section{SHP2}

C
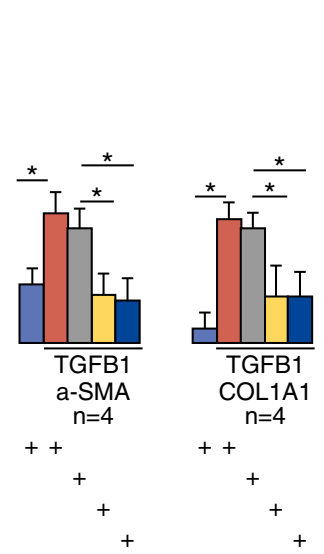

E76A

TGFB1
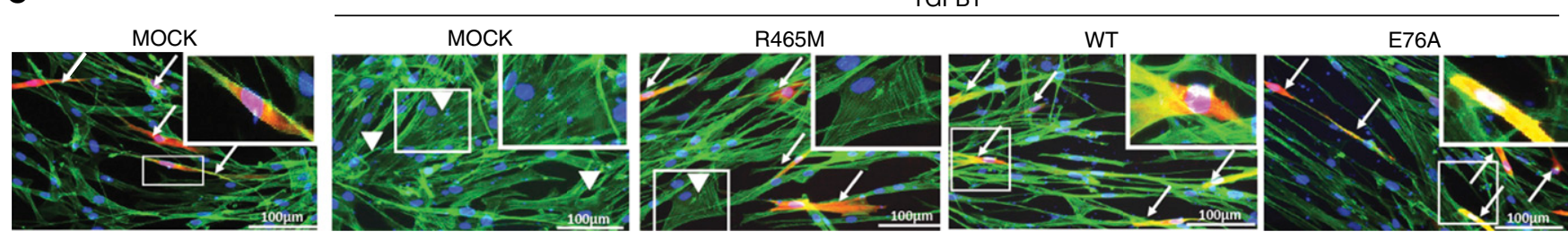

Phalloidin green/SHP2 (red)/DAPI/merged (yellow)

Figure 4. SH2 domain-containing phosphatase-2 (SHP2) suppresses transforming growth factor- $\beta_{1}$ (TGFB1)-induced myofibroblast differentiation. (A) Relative change in SHP2, $\alpha$-smooth muscle actin ( $\alpha$-SMA), and collagen type I $\alpha 1$ (COL1A1) mRNA levels at baseline and after TGF- $\beta_{1}$ stimulation. Each bar and error bar represents the mean \pm SD expression of four normal human lung fibroblast (NHLF) samples (biological replicates). Bars are shown for the relative changes (fold) by setting the indicated control level to 1 . One-way analysis of variance, ${ }^{\star} P<0.05$. (B) Immunoblot analyses of SHP2, $\alpha$-SMA, and COL1A1. Each lane represents an individual NHLF sample. Blots were stripped and reblotted, using an anti- $\beta$-actin antibody as loading control. Thin black line indicates that the lanes were run on two different gels. (C) Double-immunofluorescence analysis of representative NHLF samples, showing an increase in TGF- $\beta_{1}$-induced stress fibers as indicated by phalloidin green (arrowheads, insets) in NHLFs receiving mock treatment or treated with inactive SHP2 mutant (R465M). Note the reduction in TGF- $\beta_{1}$-induced stress fibers in cells overexpressing active SHP2 (wild-type [WT] and E76A) compared with cells overexpressing inactive SHP2 mutant (R465M) (merged yellow, arrows, insets). Boxed regions are shown enlarged as insets on the right.

major profibrotic stimuli (TGF- $\beta_{1}$, PDGF-BB) in vitro. SHP2 $2^{\mathrm{D} 61 \mathrm{G} /+}$ MLFs displayed significantly increased basal PTP activity compared with $S H P 2^{+/+}$MLFs (Figure 9A). SHP2 $2^{\mathrm{D} 61 \mathrm{G} /+}$ MLFs exhibited significant reductions in TGF- $\beta_{1}-$ mediated inductions of COL1A1 (fourfold), COL3A1 (fourfold), $\alpha$-SMA (2.6-fold), and FN1 (2.4-fold) mRNA levels (Figure 9B), as well as $\alpha$-SMA and COL1A1 protein levels (Figure 9C). TGF- $\beta_{1}$ caused less stress fiber formation and $\alpha$-SMA production in $\mathrm{SHP} 2^{\mathrm{D} 61 \mathrm{G} /+}$ MLFs compared with $\mathrm{SHP} 2^{+/+}$MLF (Figure 9E, and Figure E6E). $S H P 2^{\mathrm{D} 61 \mathrm{G} /+}$ MLFs exhibited attenuated PDGF-BB-induced and TGF- $\beta_{1}$-induced phosphorylation of c-Abl and Smad2/3, respectively.

Figure 3. (Continued). decrease in PDGF-BB-induced proliferation (1.2-fold) compared with mock- or R465M-treated cells. Each bar represents mean expression of six NHLF samples (biological replicates). Data (ratio of $24 \mathrm{~h}$ [black bar portion] to $0 \mathrm{~h}$ [gray bar portion], absorbance) represent means \pm SD. One-way analysis of variance (ANOVA), ${ }^{*} P<0.05$. (B) NHLFs were treated with the indicated plasmids without growth factor stimulation, and cells were harvested 24 hours afterward for RNA extraction. Shown is the relative change in SHP2, caspase-3 (CASP3), and B-cell CLL/lymphoma 2 (BCL2) mRNA levels. Each bar and error bar represents the mean \pm SD expression of four samples (biological replicates). Bars are shown for the relative changes (fold) by setting the indicated control level to 1.0. One-way ANOVA, ${ }^{\star} P<0.05$. (C) NHLFs were treated with the indicated plasmids, and cells were harvested 48 hours afterward for protein extraction. Shown are immunoblot analyses of SHP2, cleaved (c)-CASP3, and BCL2. Each lane represents an individual NHLF sample. Vertical black line indicates that the lanes were run on two different gels. (D) Caspase-3/7 activity in NHLFs treated with the indicated plasmids for 48 hours. Data are normalized to caspase-3/7 activity (median absorbance) observed in R465M-treated cells. Bars and error bars represent mean \pm SD activity of four NHLF samples (biological replicates). (E) Immunofluorescence analysis for terminal deoxynucleotidyltransferase dUTP nick end labeling (TUNEL)-positive cells after transfection with the indicated plasmids for 48 hours. Samples that were incubated with only the labeling solution were used as negative controls. (F) Quantitative scoring of TUNEL/DAPI (4',6-diamidino-2-phenylindole) double-positive cells as a percentage of total DAPI-positive cells from four NHLF samples (biological replicates). Data represent means \pm SD; one-way ANOVA, ${ }^{\star} P<0.05$. (G) SHP2 protein tyrosine phosphatase (PTP) activity in NHLFs transfected with the indicated plasmids for 48 hours. Bars and error bars represent mean $\pm \mathrm{SD}$ (nmol/min) activity of two NHLF samples (biological replicates). 
A

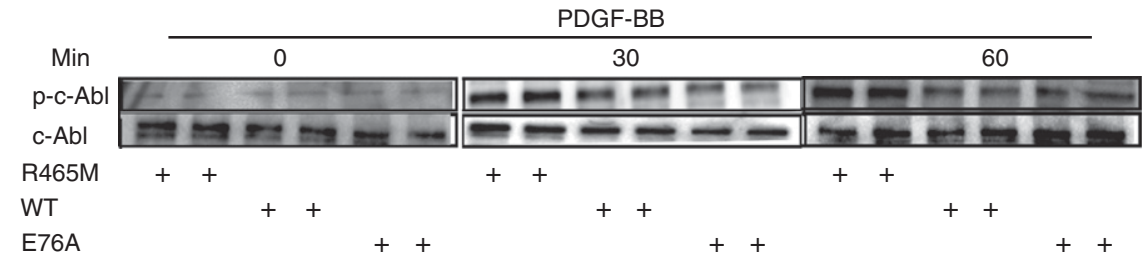

B

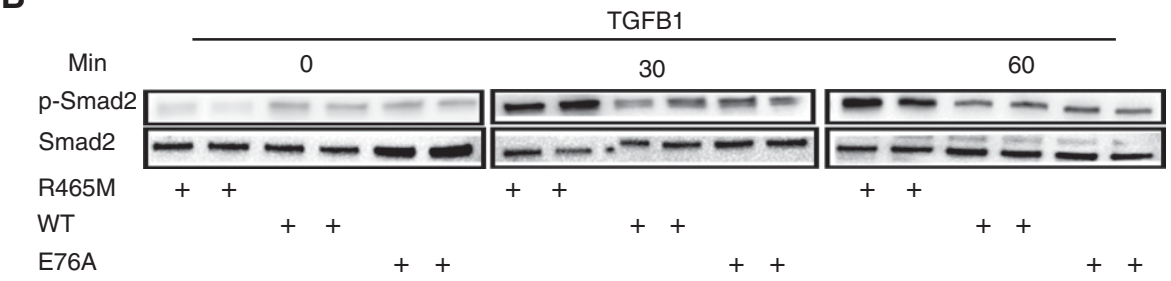

C

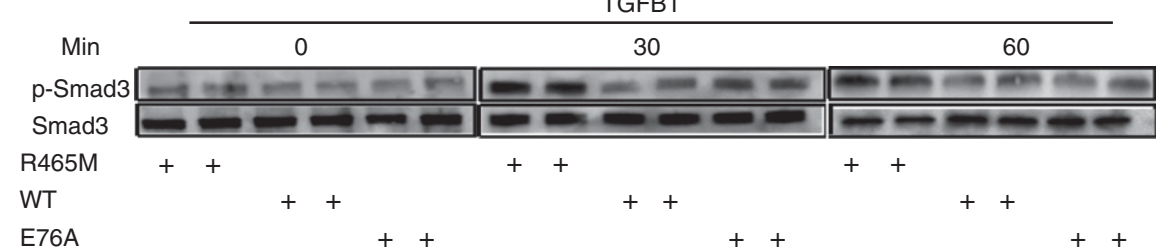

Figure 5. SH2 domain-containing phosphatase-2 (SHP2) negatively regulates phosphorylation states of tyrosine kinase and serine/threonine kinase fibrotic pathways. $(A)$ Immunoblot analyses for phosphorylated (p)-c-Abl and total c-Abl at various time points (0,30, $60 \mathrm{~min})$ after platelet-derived growth factor (PDGF)-BB stimulation. Each lane represents an individual normal human lung fibroblast (NHLF) preparation. (B) Immunoblot analyses for $\mathrm{p}$-Smad2 and total Smad2.

(C) Immunoblot analyses for $\mathrm{p}$-Smad3 and total Smad3. Note the significant attenuation in the PDGFBB- and transforming growth factor- $\beta_{1}$ (TGFB1)-induced c-Abl and Smad2/3 phosphorylation in NHLFs treated with active SHP2 (wild type [WT], E76A) compared with the inactive SHP2 mutant (R465M).

\section{Discussion}

Our study suggests a novel role for SHP2 down-regulation in pulmonary fibrosis through loss of its modulatory effects on fibroblasts. After the novel observation that SHP2 is preferentially decreased in IPF lung fibroblasts, we focused on the role of SHP2 in fibroblasts. We discovered that SHP2 catalytic activity was required to sustain steady state fibroblast differentiation and that reduction in the level or activity of SHP2 was sufficient to induce fibroblast-to-myofibroblast differentiation in primary human lung fibroblasts, as well as to increase their sensitivity to profibrotic stimuli. Overexpression of SHP2 or constitutively active mutants of SHP2 reduced the responsiveness of primary lung fibroblasts to profibrotic stimuli by dephosphorylation of major kinase-controlled profibrotic signaling pathways. Phenotypic analysis of primary lung fibroblasts obtained from mice heterozygous for the Noonan syndrome-associated SHP2 active mutant $\left(\mathrm{SHP} 2^{\mathrm{D} 61 \mathrm{G} /+}\right)$ further confirmed the specific role of SHP2 in maintenance of fibroblast homeostasis. In vivo inhibition of SHP2 catalytic activity led to enhanced murine lung fibrosis, mice heterozygous for the Noonan syndrome-associated SHP2 active mutant $\left(S H P 2^{\mathrm{D} 61 \mathrm{G} /+}\right)$ were relatively resistant to bleomycin-induced lung fibrosis, and therapeutic lentiviral overexpression of SHP2 blunted bleomycin-induced pulmonary fibrosis, supporting a role for augmentation of SHP2 expression and activity as a therapeutic option in pulmonary fibrosis.

SHP2 is a ubiquitously expressed protein tyrosine phosphatase that plays a fundamental role in development and cell homeostasis $(34,35)$. Global SHP2 deficiency is embryonically lethal in mice, due to major developmental defects (33, 36). Support for a potential role for SHP2 in lung fibrosis has emerged from observations that targeted genetic ablations of SHP2 in AECs (19) or macrophages (20) are profibrotic. However, these studies did not assess the expression of SHP2 in human pulmonary fibrosis. When we analyzed tissues obtained from patients with IPF we discovered that SHP2 expression was preferentially decreased in myofibroblast foci. To study the consequences of SHP2 down-regulation in fibroblasts we employed transient gene knockdown, using siRNA, and catalytic domain inhibition, using PHPS1. Both SHP2 knockdown and SHP2 chemical inhibition caused spontaneous fibroblastto-myofibroblast differentiation as assessed by increased formation of stress fibers and up-regulation of fibrotic markers, suggesting that SHP2 expression and catalytic activity are required for maintenance of fibroblast differentiation. Our findings are consistent with earlier observations showing that fibroblasts lacking a functional SHP2 (37) or SHP2-knockdown mouse embryonic fibroblasts (17) exhibit increased formation of stress fibers. We complemented these observations by performing in vitro SHP2 gain-of-function experiments. Overexpression of both the wild-type SHP2 and an active mutant of SHP2 reduced their sensitivity to profibrotic stimuli and prevented TGF$\beta_{1}$-induced fibroblast-to-myofibroblast differentiation. These in vitro results were augmented by in vivo observations that inhibition of SHP2 activity was profibrotic and that SHP2 augmentation blunted bleomycin-induced lung fibrosis. Taken together with the observations in the IPF lung, our results suggest a novel and critically important role for SHP2 activity in fine-tuning regulation of fibroblast phenotypes in pulmonary fibrosis.

The pathways by which SHP2 mediates its antifibrotic activity are of significant interest. We provide significant evidence that SHP2 affects both the PDGF-BB and TGF- $\beta_{1}$ pathways (Figure E7). Increased expression of SHP2 or of a constitutively active mutant of SHP2 was sufficient to sustain fibroblast homeostasis and blunted the response to profibrotic stimuli through attenuated PDGF-BBmediated $\mathrm{c}-\mathrm{Abl}$ phosphorylation. This is in line with earlier in vitro data identifying endogenous Abl kinases as SHP2 substrates during PDFGBB-mediated fibroblast proliferation (18). Less clear is the observation that both 
A

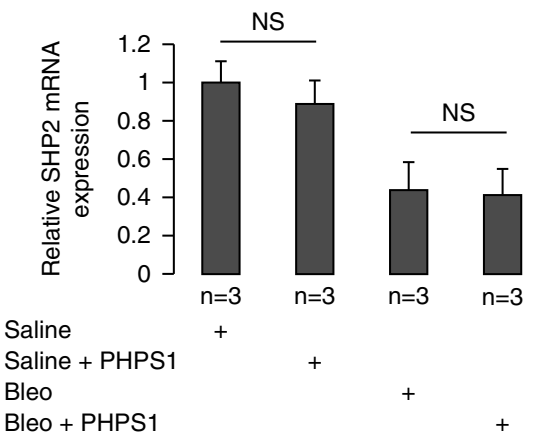

B

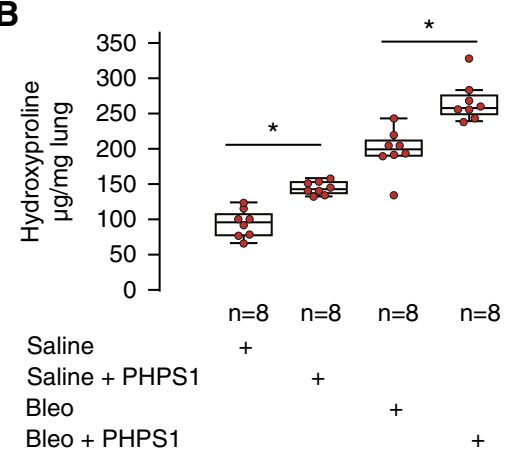

C
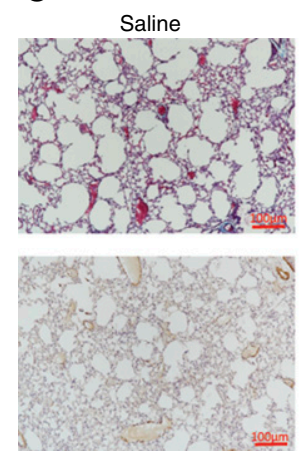

Saline + PHPS1
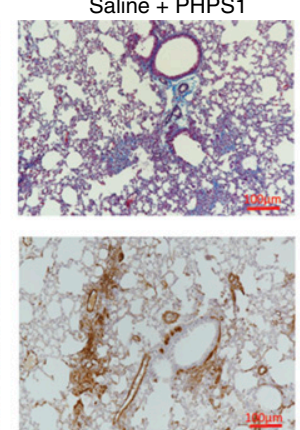
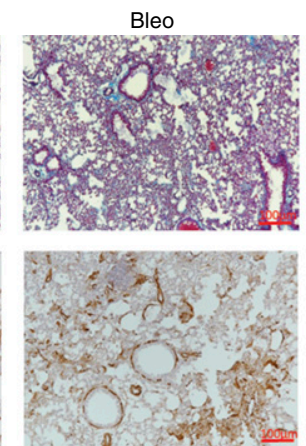
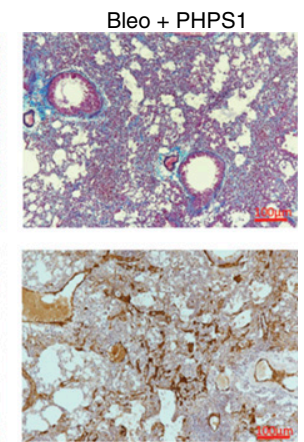

Figure 6. $\mathrm{SH} 2$ domain-containing phosphatase-2 (SHP2) catalytic inhibition aggravates bleomycin (Bleo)-induced lung fibrosis. (A) Relative change in SHP2 mRNA levels after treatment with saline or bleomycin with or without phenylhydrazonopyrazolone sulfonate 1 (PHPS1) and phosphate-buffered saline (PBS). PHPS1 administration exerted nonsignificant (NS) changes in SHP2 mRNA levels within saline and bleomycin treatment groups. Each bar and error bar represents mean \pm SD expression of three samples (biological replicates). Bars are shown for the relative changes (fold) by setting the indicated control level to 1.0. One-way analysis of variance, ${ }^{\star} P<0.05$. (B) Increased collagen deposition (assessed by hydroxyproline content) in saline-challenged mice treated with PHPS1 compared with those treated with PBS (142.5 vs. 95.6 [interquartile range, 137.1-152.1 vs. 77.5-107.3], respectively). Hydroxyproline levels were significantly elevated in bleomycin-challenged mice treated with PHPS1 compared with those treated with PBS (257.5 vs. 198.9 [interquartile range, 248.9-275.6 vs. 190.4-211.7], respectively). Data ( $\mu \mathrm{g} / \mathrm{mg}$ right lung) are presented as box-and-whisker plots, with horizontal bars representing medians. Mann-Whitney $U$ test for independent samples, ${ }^{\star} P<0.05$.

(C) Masson's trichrome (upper panel) or $\alpha$-smooth muscle actin (lower panel) staining of representative lung sections $(n=3)$ from each group of treated mice. Increased collagen deposition can be seen in the interstitium, peribronchiolar space, or perivascular space in PHPS1challenged compared with PHPS1-unchallenged mice.

transient expression of SHP2 and the Noonan syndrome-associated constitutively active mutant of SHP2 suppressed TGF- $\beta_{1}$-mediated Smad2/3 phosphorylation. SHP2 is a tyrosine phosphatase and unlikely to directly dephosphorylate the serine/threonine site on Smad3. However, it has been shown that the nonreceptor tyrosine kinase $\mathrm{c}-\mathrm{Abl}$ partly mediates the signaling events of the TGF- $\beta_{1}$-induced serine/threonine kinase pathway (38). Therefore, it can be speculated that the SHP2-induced deactivation of Smad2/3 could be the consequence of direct SHP2 phosphatase-dependent dephosphorylation of c-Abl. Our results are consistent with observations that SHP2 loss from AECs promotes epithelial-mesenchymal transition and fibrotic growth (19) but differ from those showing that SHP2 overexpression positively regulates TGF$\beta_{1}$-induced airway remodeling in bronchial epithelial cells (39). Although the reasons for this discrepancy are unclear, it is possible that SHP2 will have different effects on different cells as has been previously proposed (33). More importantly, in the context of current U.S. Food and Drug Administrationapproved therapies, the emergence of SHP2 as a potential modulator of TGF- $\beta_{1}$ - and PDGF-BB-mediated fibrotic pathways, as well as our findings that expression of a constitutively active SHP2 mutant or lung-targeted lentiviral administration of SHP2 blunt pulmonary fibrosis, are of significant interest. It has been previously suggested that nintedanib is a phosphatase activator (32) and we observed that nintedanib restores PDGF-BB-mediated down-regulation of SHP2 expression and activity in NHLFs (Figure E8). It is tempting to hypothesize that part of the effect of nintedanib is mediated through induction of SHP2, and thus it would be most effective in patients with low SHP2 activity.

Our data exhibit a number of limitations. The present study was not designed to identify the causes for SHP2 deficiency in IPF lungs. Our in vitro and in vivo data suggest that SHP2 downregulation is induced by profibrotic stimuli (TGF- $\beta_{1}$, PDGF-BB) or injury (bleomycin). Although the exact stimuli in the human lung are unknown, a role for the same profibrotic cytokines is suggested in human IPF, and it could be that these cytokines drive SHP2 downregulation in the IPF lung. However, it is possible that epigenetic modifications in the promoter region of SHP2, as happens in patients with leukemia (40), or effects of chronically enhanced changes in matrix stiffness, may affect SHP2 expression and could drive fibroblast-specific downregulation in humans. Similarly, the exact mechanisms underlying the therapeutic effects of SHP2-lentiviral restoration in the bleomycin model of lung fibrosis are still elusive. We hypothesize that SHP2 overexpression or inhibition promotes significant alterations in the phosphorylation states of several proteins involved in major signal transduction pathways. Because of the ubiquitous expression of SHP2 and its multitude of effects, it is possible that SHP2 inhibition may alter the general phosphorylation/dephosphorylation state of multiple signal transduction pathways in other cell types, not only 
A

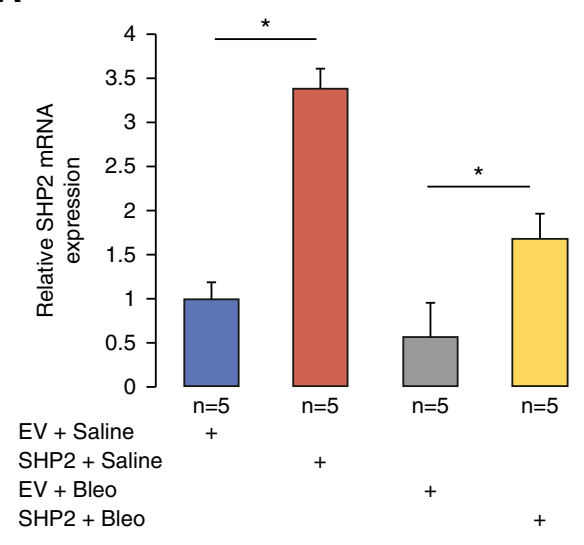

D

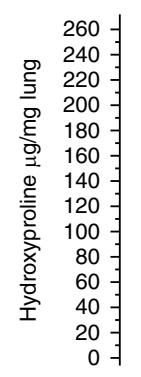

$\mathrm{EV}+$ Saline

SHP2 + Saline

$\mathrm{EV}+\mathrm{Bleo}$

SHP2 + Bleo
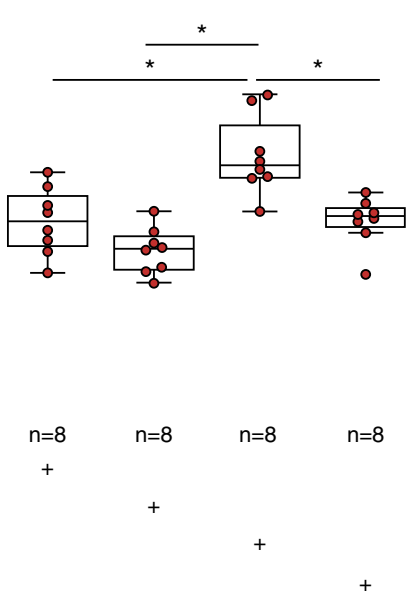

B

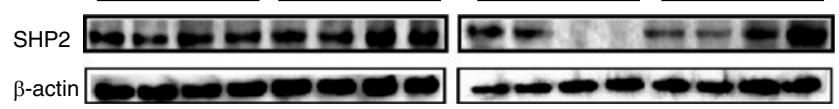

C

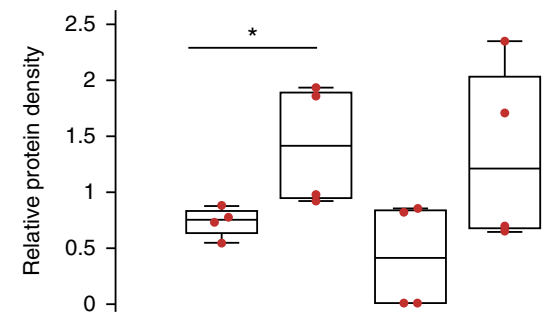

$\mathrm{EV}+$ Saline

SHP2 + Saline

$\mathrm{EV}+\mathrm{Bleo}$

SHP2 + Bleo

E
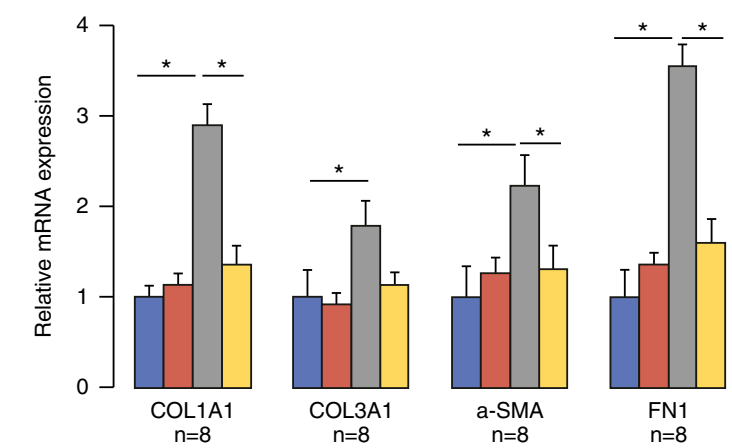

EV + Saline +
SHP2 + Saline +
EV + Bleo
SHP2 + Bleo
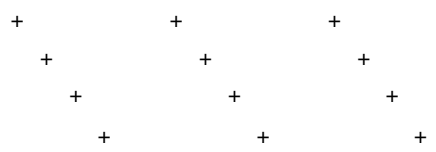

\section{$\mathbf{F}$}
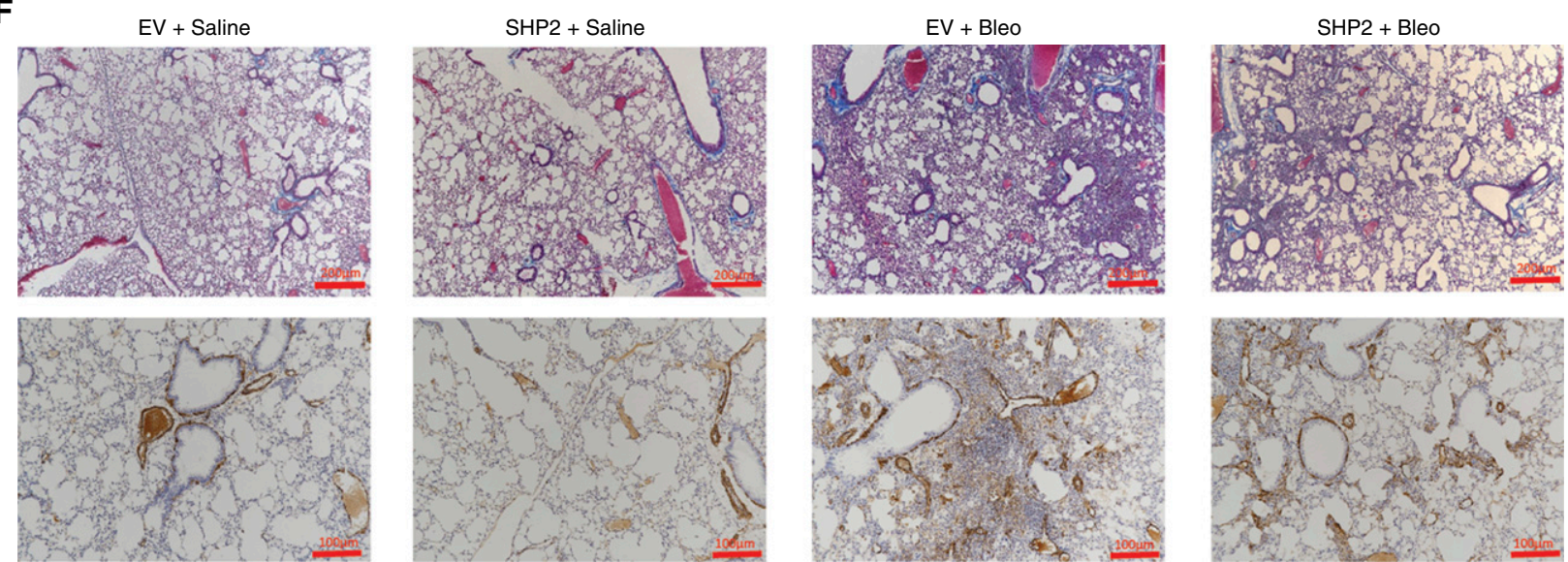

Figure 7. Lung-targeted lentiviral administration of SH2 domain-containing phosphatase-2 (SHP2) attenuates bleomycin (Bleo)-induced lung fibrosis. (A) Relative change in SHP2 mRNA levels after treatment with SHP2-lentivector or empty vector (EV) followed by bleomycin or saline challenge. Each bar and error bar represents mean \pm SD expression of five samples (biological replicates). Bars are shown for the relative changes (fold) by setting the indicated control level to 1.0. One-way analysis of variance (ANOVA), ${ }^{*} P<0.05$. (B) Immunoblot analyses for SHP2 levels in lenti-SHP2- or EV-treated animals stimulated with bleomycin or saline. Each lane represents a mouse lung sample. (C) Densitometry immunoblot analyses for SHP2 levels normalized to $\beta$-actin levels revealed increased SHP2 expression in mouse lungs challenged with saline and infected with SHP2-lentivector compared with EV-infected lungs (1.4 vs. 0.8; interquartile range [IQR], 0.9-1.9 vs. 0.6-0.8). A trend toward increased expression was also noticed in the SHP2-lentivector mice challenged with bleomycin compared with EV-infected mice, although it was not statistically significant (1.2 vs. 0.4; IQR, 0.7-2.0 vs. 0.01-0.8). Data are 
A

A
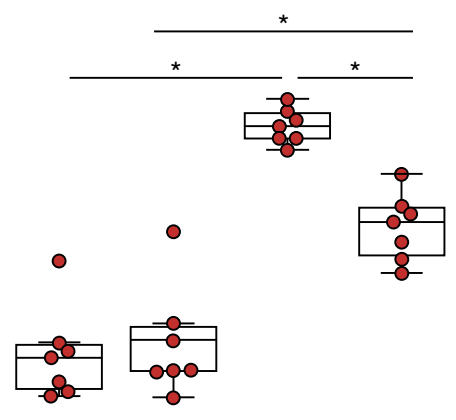

$\mathrm{n}=7 \quad \mathrm{n}=7$

$n=7$

$\mathrm{n}=7$

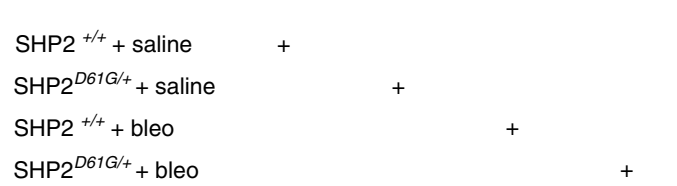

B

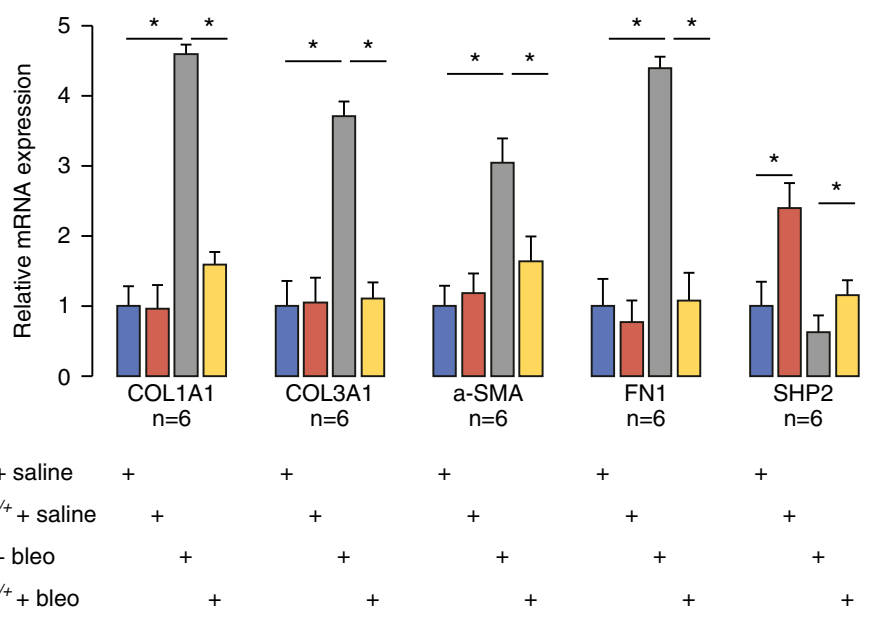

\section{C}
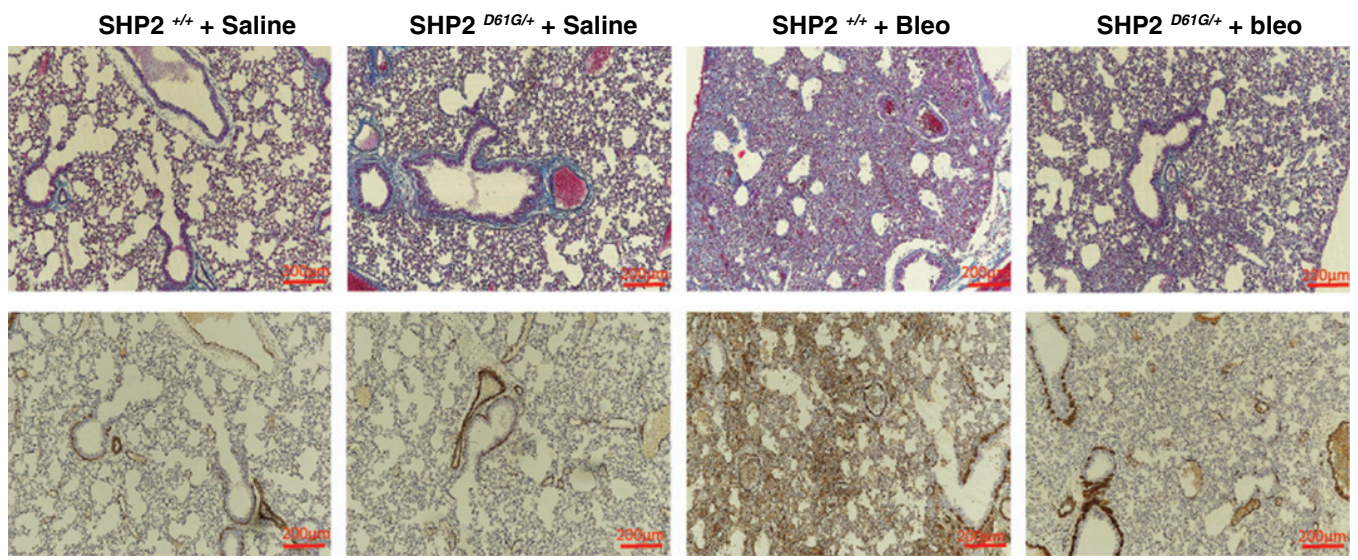

Figure 8. The Noonan syndrome-associated SH2 domain-containing phosphatase-2 active mutant (SHP2 ${ }^{\mathrm{D} 61 \mathrm{G} /{ }^{+}}$) confers resistance to bleomycin (Bleo)induced pulmonary fibrosis. (A) Decreased collagen deposition (assessed by hydroxyproline content) after bleomycin challenge in SHP2 ${ }^{\mathrm{D} 61 \mathrm{G} /+}$ mice compared with wild-type littermates (182.5 vs. 134.9; interquartile range, 176.7-188.8 vs. 118.3-141.8). Data ( $\mu \mathrm{g} / \mathrm{mg}$ right lung) are presented as boxand-whisker plots, with horizontal bars representing medians. Mann-Whitney $U$ test for independent samples, ${ }^{*} P<0.05$. (B) Relative change in collagen type I $\alpha 1$ (COL1A1), collagen type III $\alpha 1$ (COL3A1), $\alpha$-smooth muscle actin ( $\alpha$-SMA), fibronectin-1 (FN1), and SHP2 mRNA levels. Each bar and error bar represents mean \pm SD expression of six samples (biological replicates). Bars are shown for the relative changes (fold) by setting the indicated control level to 1.0. One-way analysis of variance, ${ }^{\star} P<0.05$. (C) Masson's trichrome (top) and $\alpha$-SMA (bottom) staining of representative lung sections ( $\mathrm{n}=4$ ) from each group of treated mice. Decreased collagen deposition and $\alpha$-SMA staining is observed in the interstitium, peribronchiolar space, or perivascular space in $\mathrm{SHP} 2^{\mathrm{D} 61 \mathrm{G} /+}$ mice compared with $\mathrm{SHP} 2^{+/+}$mice.

fibroblasts, thus making the lung, as an organ, more susceptible to fibrosis, as has been previously described $(19,20)$. This does not reduce the significant value of our observation that overexpression of catalytically active SHP2 has the potential to blunt fibrosis. However, it may suggest that additional experiments, including dissection of the contribution of SHP2 signaling in various cell types and detailed identification of all SHP2 targets,

Figure 7. (Continued). presented as box-and-whisker plots, with horizontal bars representing medians. Mann-Whitney $U$ test for independent samples, ${ }^{\star} P<0.05$. (D) Decreased collagen deposition (assessed by hydroxyproline content) after bleomycin challenge in mice treated with SHP2-lentivector compared with EV-treated mice (153.1 vs. 193.5; IQR, 144.6-159.8 vs. $183.8-224.8$, respectively). Data ( $\mu$ g/mg right lung) are presented as box-and-whisker plots, with horizontal bars representing medians. Mann-Whitney $U$ test for independent samples, ${ }^{\star} P<0.05$. (E) Relative change in collagen type I $\alpha 1$ (COL1A1), collagen type III $\alpha 1$ (COL3A1), $\alpha$-smooth muscle actin ( $\alpha$-SMA), and fibronectin-1 (FN1) levels. Each bar and error bar represents mean \pm SD expression of five samples (biological replicates). Bars are shown for the relative changes (fold) by setting the indicated control level to 1.0. One-way ANOVA, ${ }^{\star} P<0.05$. ( $F$ ) Masson's trichrome (top) and $\alpha$-SMA (bottom) staining of representative lung sections $(\mathrm{n}=3)$ from each group of treated mice. Decreased collagen and $\alpha$-SMA deposition are observed in the interstitium, peribronchiolar space, or perivascular space in SHP2-treated mice compared with untreated mice. 
A

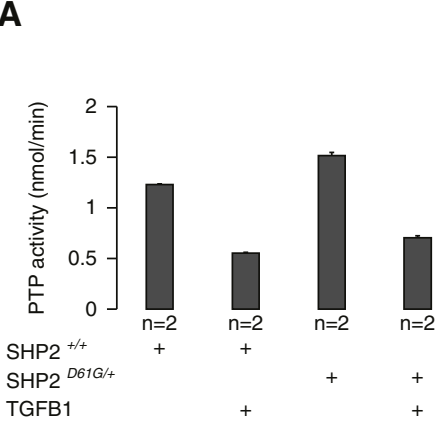

B

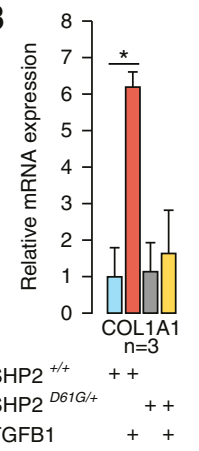

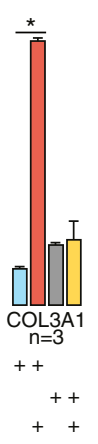

C

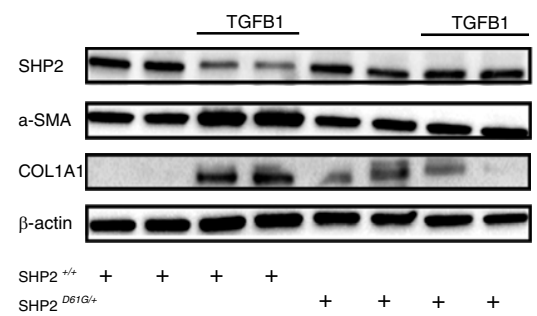

$\mathrm{SHP} 2^{+/+}+\mathrm{TGFB} 1$

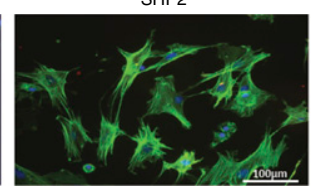

SHP2 ${ }^{D 616 / 4}$
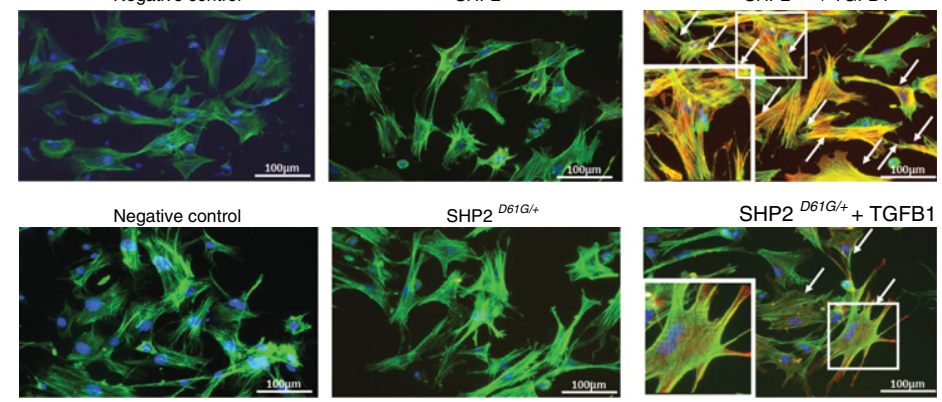

SHP2 ${ }^{D 61 G / 4}+$ TGFB1

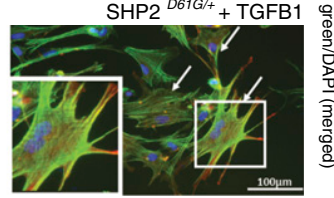

E
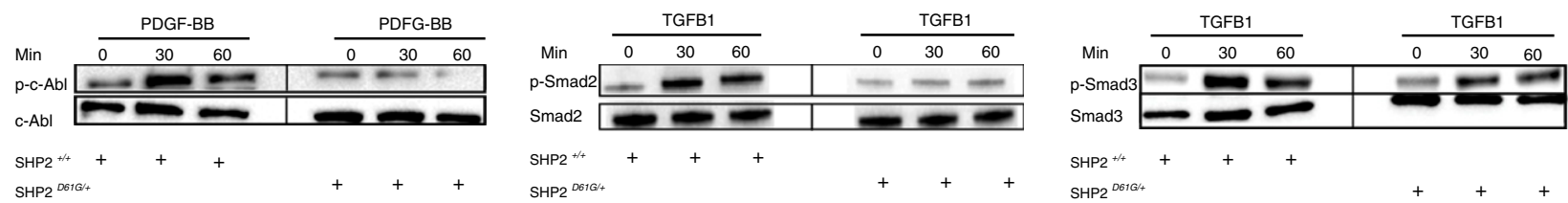

Figure 9. Primary mouse lung fibroblasts expressing the Noonan syndrome-associated SH2 domain-containing phosphatase-2 active mutant $\left(\mathrm{SHP} 2^{\mathrm{D} 61 \mathrm{G} /+}\right.$ ) display attenuated fibrotic responses in vitro. (A) SHP2 protein tyrosine phosphatase (PTP) activity in SHP2 ${ }^{\mathrm{D} 61 \mathrm{G} /+}$ mutant and $\mathrm{SHP}^{+/+}$mouse lung fibroblasts. Bars and error bars represent mean $\pm \mathrm{SD}$ (nmol/min) activity of two normal human lung fibroblast samples (biological replicates). (B) Relative change in collagen type I $\alpha 1$ (COL1A1), collagen type III $\alpha 1$ (COL3A1), $\alpha$-smooth muscle actin ( $\alpha$-SMA), fibronectin-1 (FN1), and SHP2 mRNA levels at baseline and after transforming growth factor (TGF)- $\beta_{1}$ stimulation. Each bar and error bar represents mean \pm SD expression of three samples in duplicate. Bars are shown for the relative changes (fold) by setting the indicated control level to 1.0. One-way analysis of variance, ${ }^{*} P<0.05$. (C) Immunoblot analyses of SHP2, $\alpha$-SMA, and COL1A1. Each lane represents an individual fibroblast sample. Blots were stripped and reblotted, using an anti- $\beta$-actin antibody as loading control. (D) Double-immunofluorescence analysis in representative mouse lung fibroblasts showing reduction in TGF- $\beta_{1}$-induced stress fibers and $\alpha$-SMA expression in SHP2 ${ }^{\mathrm{D} 61 \mathrm{G} /+}$ mutant lung fibroblasts compared with TGF- $\beta_{1}-$ stimulated SHP2 ${ }^{+/+}$lung fibroblasts, as indicated by colocalization of $\alpha$-SMA (red) with filamentous actin (phalloidin green) (merged yellow cells shown by arrows). Samples that were incubated without the primary antibody ( $\alpha$-SMA) were used as negative controls. Boxed regions are shown enlarged as insets on the left. (E) Immunoblot analyses for phosphorylated ( $p$ )-c-Abl and total c-Abl (left), p-Smad2, and total Smad2 (middle), and p-Smad3 and total Smad3 (right) at various time points (0, 30, 60 min) after platelet-derived growth factor (PDGF)-BB $(25 \mathrm{ng} / \mathrm{ml})$ and TGF- $\beta_{1}(10 \mathrm{ng} / \mathrm{ml})$ stimulation, respectively. Each lane represents an individual sample preparation. Thin black line indicates that all the lanes derived from the same blot but were noncontiguous and were cut and pasted as joint images. Note the significant attenuation in the PDGF-BB- and TGF- $\beta_{1}$-induced c-Abl and Smad2/3 phosphorylation in SHP2 ${ }^{\mathrm{D} 61 \mathrm{G} /+} \mathrm{Compared}^{\mathrm{cith}} \mathrm{SHP} 2^{+/+}$ lung fibroblasts.

will be required to decipher the therapeutic mechanism of SHP2 in lung fibrosis and allow us to discover additional cell-specific targets downstream of SHP2 signaling. In this context it is important to highlight that SHP2 augmentation is not without risk. In humans gain-offunction SHP2 mutations are associated with developmental disorders, the most well known being the Noonan syndrome (41). Although most of the phenotypic aspects of the syndrome are associated with developmental defects induced by SHP2 activation during specific stages of embryonic and postnatal development, it seems logical to suggest that strategies aimed at activating SHP2 will be targeted to the lung. Like any other drug, detailed safety and efficacy studies will be required.
In conclusion, our present study reveals an important and previously unrecognized role of SHP2 as master regulator of fibroblast homeostasis and provides significant data to support its consideration as a therapeutic target in IPF. Loss of SHP2 expression and activity leads to enhanced fibrotic responses in both fibroblast cell lines and animal models of lung fibrosis. We provide significant 
evidence that restoring bleomycin-induced SHP2 down-regulation, using lentivirus in vivo, or genetically enhancing SHP2 activity, using a constitutively active mutant, attenuates fibrotic changes in a murine model of pulmonary fibrosis. Taken together with our observations that SHP2 is specifically down-regulated in myofibroblast foci in IPF, our results suggest that augmenting SHP2 activity should be evaluated as a novel and potentially effective antifibrotic therapy for patients with IPF.
Author disclosures are available with the text of this article at www.atsjournals.org.

Acknowledgment: The authors thank Dr. Carol Feghali-Bostwick (Division of Rheumatology and Immunology, Medical University of South Carolina) for providing idiopathic pulmonary fibrosis and normal human lung fibroblasts.

\section{References}

1. Raghu G, Collard HR, Egan JJ, Martinez FJ, Behr J, Brown KK, Colby TV, Cordier JF, Flaherty KR, Lasky JA, et al.; ATS/ERS/JRS/ ALAT Committee on Idiopathic Pulmonary Fibrosis. An official ATS/ERS/JRS/ALAT statement: idiopathic pulmonary fibrosis: evidence-based guidelines for diagnosis and management. Am J Respir Crit Care Med 2011;183:788-824.

2. Raghu G, Rochwerg B, Zhang Y, Garcia CA, Azuma A, Behr J, Brozek JL, Collard HR, Cunningham W, Homma S, et al.; American Thoracic Society; European Respiratory Society; Japanese Respiratory Society; Latin American Thoracic Association. An official ATS/ERS/JRS/ALAT clinical practice guideline: treatment of idiopathic pulmonary fibrosis: an update of the 2011 clinical practice guideline. Am J Respir Crit Care Med 2015;192:e3-e19. [Published erratum appears in Am J Respir Crit Care Med 192:644.]

3. Rosas IO, Kaminski N. Update in diffuse parenchymal lung disease, 2013. Am J Respir Crit Care Med 2015;191:270-274.

4. Selman M, Buendía-Roldán I, Pardo A. Aging and pulmonary fibrosis. Rev Invest Clin 2016;68:75-83.

5. Fernandez IE, Eickelberg O. New cellular and molecular mechanisms of lung injury and fibrosis in idiopathic pulmonary fibrosis. Lancet 2012; 380:680-688.

6. Fingerlin TE, Murphy E, Zhang W, Peljto AL, Brown KK, Steele MP, Loyd JE, Cosgrove GP, Lynch D, Groshong S, et al. Genome-wide association study identifies multiple susceptibility loci for pulmonary fibrosis. Nat Genet 2013;45:613-620.

7. Seibold MA, Wise AL, Speer MC, Steele MP, Brown KK, Loyd JE, Fingerlin TE, Zhang W, Gudmundsson G, Groshong SD, et al. A common MUC5B promoter polymorphism and pulmonary fibrosis. N Engl J Med 2011;364:1503-1512.

8. Daccord C, Maher TM. Recent advances in understanding idiopathic pulmonary fibrosis. F1000Res 2016;5.

9. King TE Jr, Bradford WZ, Castro-Bernardini S, Fagan EA, Glaspole I, Glassberg MK, Gorina E, Hopkins PM, Kardatzke D, Lancaster L, et al.; ASCEND Study Group. A phase 3 trial of pirfenidone in patients with idiopathic pulmonary fibrosis. N Engl J Med 2014;370: 2083-2092.

10. Richeldi L, du Bois RM, Raghu G, Azuma A, Brown KK, Costabel U, Cottin V, Flaherty KR, Hansell DM, Inoue Y, et al.; INPULSIS Trial Investigators. Efficacy and safety of nintedanib in idiopathic pulmonary fibrosis. N Engl J Med 2014;370:2071-2082.

11. Grimminger F, Günther A, Vancheri $C$. The role of tyrosine kinases in the pathogenesis of idiopathic pulmonary fibrosis. Eur Respir J 2015; 45:1426-1433.

12. Ostman A, Böhmer FD. Regulation of receptor tyrosine kinase signaling by protein tyrosine phosphatases. Trends Cell Biol 2001;11:258-266.

13. Lee H, Bennett AM. Receptor protein tyrosine phosphatase-receptor tyrosine kinase substrate screen identifies EphA2 as a target for LAR in cell migration. Mol Cell Biol 2013;33:1430-1441.

14. Chan RJ, Feng GS. PTPN11 is the first identified proto-oncogene that encodes a tyrosine phosphatase. Blood 2007;109:862-867.

15. Fragale A, Tartaglia M, Wu J, Gelb BD. Noonan syndrome-associated SHP2/PTPN11 mutants cause EGF-dependent prolonged GAB1 binding and sustained ERK2/MAPK1 activation. Hum Mutat 2004;23: 267-277.

16. Paardekooper Overman J, Yi JS, Bonetti M, Soulsby M, Preisinger C, Stokes MP, Hui L, Silva JC, Overvoorde J, Giansanti P, et al. PZR coordinates Shp2 Noonan and LEOPARD syndrome signaling in zebrafish and mice. Mol Cell Biol 2014;34:2874-2889.
17. Nakata S, Fujita N, Kitagawa Y, Okamoto R, Ogita H, Takai Y. Regulation of platelet-derived growth factor receptor activation by afadin through SHP-2: implications for cellular morphology. J Biol Chem 2007;282:37815-37825.

18. Mitra S, Beach C, Feng GS, Plattner R. SHP-2 is a novel target of Abl kinases during cell proliferation. J Cell Sci 2008;121:3335-3346.

19. Zhang X, Zhang Y, Tao B, Teng L, Li Y, Cao R, Gui Q, Ye M, Mou X, Cheng $\mathrm{H}$, et al. Loss of Shp2 in alveoli epithelia induces deregulated surfactant homeostasis, resulting in spontaneous pulmonary fibrosis. FASEB J 2012;26:2338-2350.

20. Tao B, Jin W, Xu J, Liang Z, Yao J, Zhang Y, Wang K, Cheng $\mathrm{H}$, Zhang X, Ke Y. Myeloid-specific disruption of tyrosine phosphatase Shp2 promotes alternative activation of macrophages and predisposes mice to pulmonary fibrosis. J Immunol 2014;193: 2801-2811.

21. Tzouvelekis A, Lino Cardenas CL, Yu G, Sakamoto K, Deluliis J, Wyllie A, Ahangari F, Woolard T, Heather L, Herazo-Maya JD, et al. Ptpn11 is a novel anti-fibrotic gene in idiopathic pulmonary fibrosis [abstract]. Am J Respir Crit Care Med 2015;191:A2356.

22. Kusko RL, Brothers li JF, Tedrow J, Pandit K, Huleihel L, Perdomo C Liu G, Juan-Guardela B, Kass D, Zhang S, et al. Integrated genomics reveals convergent transcriptomic networks underlying chronic obstructive pulmonary disease and idiopathic pulmonary fibrosis. Am J Respir Crit Care Med 2016;194:948-960.

23. Kim S, Herazo-Maya JD, Kang DD, Juan-Guardela BM, Tedrow J, Martinez FJ, Sciurba FC, Tseng GC, Kaminski N. Integrative phenotyping framework (iPF): integrative clustering of multiple omics data identifies novel lung disease subphenotypes. BMC Genomics 2015;16:924.

24. Bauer Y, Tedrow J, de Bernard S, Birker-Robaczewska M, Gibson KF, Guardela BJ, Hess P, Klenk A, Lindell KO, Poirey S, et al. A novel genomic signature with translational significance for human idiopathic pulmonary fibrosis. Am J Respir Cell Mol Biol 2015;52:217-231.

25. Kontaridis MI, Liu X, Zhang L, Bennett AM. Role of SHP-2 in fibroblast growth factor receptor-mediated suppression of myogenesis in C2C12 myoblasts. Mol Cell Biol 2002;22:3875-3891.

26. Eminaga S, Bennett AM. Noonan syndrome-associated SHP-2/Ptpn11 mutants enhance SIRP $\alpha$ and PZR tyrosyl phosphorylation and promote adhesion-mediated ERK activation. J Biol Chem 2008;283: 15328-15338.

27. Pilewski JM, Liu L, Henry AC, Knauer AV, Feghali-Bostwick CA. Insulinlike growth factor binding proteins 3 and 5 are overexpressed in idiopathic pulmonary fibrosis and contribute to extracellular matrix deposition. Am J Pathol 2005;166:399-407.

28. Zhang Y, Jiang G, Sauler M, Lee PJ. Lung endothelial HO-1 targeting in vivo using lentiviral miRNA regulates apoptosis and autophagy during oxidant injury. FASEB $J$ 2013;27:4041-4058.

29. Wu W, Dave N, Tseng GC, Richards T, Xing EP, Kaminski N. Comparison of normalization methods for CodeLink Bioarray data. BMC Bioinformatics 2005;6:309.

30. Hellmuth K, Grosskopf S, Lum CT, Würtele M, Röder N, von Kries JP, Rosario M, Rademann J, Birchmeier W. Specific inhibitors of the protein tyrosine phosphatase Shp2 identified by high-throughput docking. Proc Natl Acad Sci USA 2008;105:7275-7280.

31. Hinz B, Gabbiani G. Mechanisms of force generation and transmission by myofibroblasts. Curr Opin Biotechnol 2003;14:538-546.

32. Morais A, Beltrão M, Sokhatska O, Costa D, Melo N, Mota P, Marques A, Delgado L. Serum metalloproteinases 1 and 7 in the diagnosis of idiopathic pulmonary fibrosis and other interstitial pneumonias. Respir Med 2015;109:1063-1068. 
33. Araki T, Mohi MG, Ismat FA, Bronson RT, Williams IR, Kutok JL, Yang W, Pao LI, Gilliland DG, Epstein JA, et al. Mouse model of Noonan syndrome reveals cell type- and gene dosagedependent effects of Ptpn11 mutation. Nat Med 2004;10: 849-857.

34. Zhang SS, Hao E, Yu J, Liu W, Wang J, Levine F, Feng GS. Coordinated regulation by Shp2 tyrosine phosphatase of signaling events controlling insulin biosynthesis in pancreatic beta-cells. Proc Natl Acad Sci USA 2009;106:7531-7536.

35. Chen B, Bronson RT, Klaman LD, Hampton TG, Wang JF, Green PJ, Magnuson T, Douglas PS, Morgan JP, Neel BG. Mice mutant for Egfr and Shp2 have defective cardiac semilunar valvulogenesis. Nat Genet 2000;24:296-299.

36. Araki T, Chan G, Newbigging S, Morikawa L, Bronson RT, Neel BG. Noonan syndrome cardiac defects are caused by PTPN11 acting in endocardium to enhance endocardial-mesenchymal transformation. Proc Natl Acad Sci USA 2009;106:4736-4741.
37. Yu DH, Qu CK, Henegariu O, Lu X, Feng GS. Protein-tyrosine phosphatase Shp-2 regulates cell spreading, migration, and focal adhesion. J Biol Chem 1998;273:21125-21131.

38. Daniels CE, Wilkes MC, Edens M, Kottom TJ, Murphy SJ, Limper AH, Leof EB. Imatinib mesylate inhibits the profibrogenic activity of TGF- $\beta$ and prevents bleomycin-mediated lung fibrosis. J Clin Invest 2004; 114:1308-1316.

39. Qin XJ, Zhang GS, Zhang X, Qiu ZW, Wang PL, Li YW, Li W, Xie QM, $\mathrm{Ke} \mathrm{YH}$, Lee JJ, et al. Protein tyrosine phosphatase SHP2 regulates TGF- $\beta_{1}$ production in airway epithelia and asthmatic airway remodeling in mice. Allergy 2012;67:1547-1556.

40. Jacob ST, Motiwala T. Epigenetic regulation of protein tyrosine phosphatases: potential molecular targets for cancer therapy. Cancer Gene Ther 2005;12:665-672.

41. Tartaglia M, Martinelli S, Stella L, Bocchinfuso G, Flex E, Cordeddu V, Zampino G, Burgt Iv, Palleschi A, Petrucci TC, et al. Diversity and functional consequences of germline and somatic PTPN11 mutations in human disease. Am J Hum Genet 2006;78:279-290. 